\title{
Promoting Sleep Oscillations and Their Functional Coupling by Transcranial Stimulation Enhances Memory Consolidation in Mild Cognitive Impairment
}

\author{
이ulia Ladenbauer, ${ }^{1,2,3 *}$ - 0 Josef Ladenbauer, ${ }^{4,5,6 *}$ Nadine Külzow, ${ }^{1,2}$ Rebecca de Boor, ${ }^{1}$ Elena Avramova, ${ }^{1}$ \\ 니rike Grittner, ${ }^{7}$ and ${ }^{\circledR}$ Agnes Flöel ${ }^{1,2,3}$ \\ ${ }^{1}$ Department of Neurology and ${ }^{2}$ NeuroCure Cluster of Excellence, Charité Universitätsmedizin Berlin, 10117 Berlin, Germany, ${ }^{3}$ Department of Neurology, \\ Universitätsmedizin Greifswald, 17475 Greifswald, Germany, ${ }^{4}$ Department of Software Engineering and Theoretical Computer Science, Technische \\ Universität Berlin, 10587 Berlin, Germany, ${ }^{5}$ Bernstein Center for Computational Neuroscience Berlin, 10115 Berlin, Germany, ${ }^{6}$ Group for Neural Theory, \\ Laboratoire de Neurosciences Cognitives, École Normale Supérieure, 75005 Paris, France, and 7Biostatistics and Clinical Epidemiology, Charité \\ Universitätsmedizin Berlin, 10117 Berlin, Germany
}

\begin{abstract}
Alzheimer's disease (AD) not only involves loss of memory functions, but also prominent deterioration of sleep physiology, which is already evident at the stage of mild cognitive impairment (MCI). Cortical slow oscillations (SO; $0.5-1 \mathrm{~Hz}$ ) and thalamocortical spindle activity $(12-15 \mathrm{~Hz})$ during sleep, and their temporal coordination, are considered critical for memory formation. We investigated the potential of slow oscillatory transcranial direct current stimulation (so-tDCS), applied during a daytime nap in a sleep-state-dependent manner, to modulate these activity patterns and sleep-related memory consolidation in nine male and seven female human patients with MCI. Stimulation significantly increased overall SO and spindle power, amplified spindle power during SO up-phases, and led to stronger synchronization between SO and spindle power fluctuations in EEG recordings. Moreover, visual declarative memory was improved by so-tDCS compared with sham stimulation and was associated with stronger synchronization. These findings indicate a well-tolerated therapeutic approach for disordered sleep physiology and memory deficits in MCI patients and advance our understanding of offline memory consolidation.
\end{abstract}

Key words: declarative memory; mild cognitive impairment; phase-amplitude coupling; sleep; transcranial electrical stimulation

Significance Statement

In the light of increasing evidence that sleep disruption is crucially involved in the progression of Alzheimer's disease (AD), sleep appears as a promising treatment target in this pathology, particularly to counteract memory decline. This study demonstrates the potential of a noninvasive brain stimulation method during sleep in patients with mild cognitive impairment (MCI), a precursor of $\mathrm{AD}$, and advances our understanding of its mechanism. We provide first time evidence that slow oscillatory transcranial stimulation amplifies the functional cross-frequency coupling between memory-relevant brain oscillations and improves visual memory consolidation in patients with MCI.

\section{Introduction}

Difficulties in forming and retrieving episodic memories are noted early in the course of Alzheimer's disease (AD) and consti-

Received Jan. 27, 2017; revised May 30, 2017; accepted June 1, 2017.

Author contributions: N.K. and A.F. designed research; Ju.L., N.K., R.d.B., and E.A. performed research; Jo.L. contributed phase-amplitude coupling analyses; Ju.L., Jo.L., and U.G. analyzed data; Ju.L., Jo.L., and A.F. wrote the paper.

This work was supported by the Deutsche Forschungsgemeinschaft (Grants FI 379-10/1, 379-11/1, SFB 910, and DFG-EXC 257) and the Bundesministerium für Bildung und Forschung (Grants FKZ 01E00801, 01GQ1424A, and 01GQ1420B). We thank Sascha Tamm for technical support, Sven Paßmann and Daria Antonenko for helpful discussions, and the subjects who participated in the study.

The authors declare no competing financial interests. tute a core component of the condition (Bäckmann et al., 2004). Likewise, sleep disturbances appear to be a major characteristic of AD-related dementia (Prinz et al., 1982) and have been reported already at the stage of mild cognitive impairment (MCI) (Westerberg et al., 2012), often a precursor of dementia due to $\mathrm{AD}$

\footnotetext{
*Ju.L. and Jo.L. contributed equally to this work.

Correspondence should be addressed to either of the following: Julia Ladenbauer, Charité Universitätsmedizin Berlin, Charitéplatz 1, 10117 Berlin, Germany. E-mail: julia.ladenbauer@charite.de. Or Agnes Flöel, Universitätsmedizin Greifswald, Ferdinand-Sauerbruch-Stra $\beta e, 17475$ Greifswald, Germany. E-mail: agnes.floeel@uni-greifswald.de.

DOI:10.1523/JNEUROSCI.0260-17.2017

Copyright $\odot 2017$ the authors $\quad 0270-6474 / 17 / 377111-14 \$ 15.00 / 0$
} 
(Sperling et al., 2011). While decline in sleep quality —including sleep parameters relevant for memory consolidation-is also common in healthy aging (Mander et al., 2013, 2014), the severity of the decline is significantly accelerated in patients with MCI or dementia due to AD (Prinz et al., 1982; Westerberg et al., 2012). Sleep disruptions not only contribute to memory deteriorations in MCI (Westerberg et al., 2012), but may also play a direct role in the progression of the underlying pathology (Wang et al., 2011; Ju et al., 2014).

Sleep plays an active role in long-term consolidation of memories (Diekelmann and Born, 2010). Specifically, slow oscillations (SO; large-amplitude waves $<1 \mathrm{~Hz})$ and sleep spindles $(8-15$ $\mathrm{Hz}$ ), which can be measured by EEG, appear to be critical for declarative memories (Schabus et al., 2004; Marshall et al., 2006). According to the "active system consolidation" account, newly encoded memories are reactivated during sleep, accompanied by sharp-wave ripple events $(80-100 \mathrm{~Hz})$ in the hippocampus, and redistributed to cortical long-term storage networks through a coordinated dialog between the hippocampus and neocortex (Rasch and Born, 2013). This dialog is mediated by a particular coupling between cortical SO and thalamocortical fast spindles $(12-15 \mathrm{~Hz})$, with spindles preferably occurring during SO upphases (Mölle et al., 2011; Ngo et al., 2013; Cox et al., 2014) and hippocampal ripples grouped at the troughs of fast spindles (Clemens et al., 2007; Staresina et al., 2015). Slow spindles (8-12 Hz) are a separate kind of sleep spindle activity whose function in memory consolidation is less well understood.

Apart from the consolidation aspect, there is mounting evidence that sleep, in particular SO, further promotes the clearance of cortical amyloid- $\beta$ (Xie et al., 2013), a peptide involved in the pathogenesis of AD (Hardy and Selkoe, 2002).

Therefore, interventions targeting sleep parameters may provide a therapeutic approach, not only for memory consolidation deficits, but also to tackle the progression of Alzheimer pathology in MCI patients (Ju et al., 2014; Mander et al., 2016), with SO activity as promising target candidate. Application of slow oscillatory weak transcranial direct current stimulation (sotDCS; frequency $<1 \mathrm{~Hz}$ ) during sleep provides a noninvasive method to enhance SO activity, as demonstrated in healthy young individuals (Marshall et al., 2006) and older individuals (Westerberg et al., 2015; Ladenbauer et al., 2016; Paßmann et al., 2016). This stimulation can also lead to increased spindle activity (Marshall et al., 2006; Ladenbauer et al., 2016; Paßmann et al., 2016), but it is not known whether it alters the cross-frequency coupling between $\mathrm{SO}$ and fast spindles, which is considered pivotal in memory consolidation during sleep. Improvements in memory due to so-tDCS have been shown repeatedly (Marshall et al., 2006; Westerberg et al., 2015; Ladenbauer et al., 2016), whereas some studies did not find any or even contrasting effects (Eggert et al., 2013; Paßmann et al., 2016).

This raises the following key questions: (1) whether and how patients with MCI who suffer from severe impairments of sleep and memory can benefit from so-tDCS during sleep in terms of SO, spindle activity, and, importantly, their cross-frequency coupling, and (2) whether so-tDCS effects are reflected behaviorally in memory performance. To address these questions, we applied so-tDCS during an afternoon nap in patients with MCI and assessed its impact on the relevant EEG-derived sleep characteristics and retention performance in a visual recognition task. Thereby we examined the potential of this noninvasive intervention for therapeutic purposes and provide novel insight on the underlying mechanisms.
Table 1. Baseline characteristics of subjects

\begin{tabular}{lc}
\hline$n$ (female/male) & $16(7 / 9)$ \\
Age (y) & $71 \pm 9(53-81)$ \\
Education (y) & $15.2 \pm 3(11-23)$ \\
Beck's Depression Index (score) & $8.6 \pm 4.2(0-14)$ \\
Mini Mental State Examination & $28.3 \pm 1.4(25-30)$ \\
AVLT, learning ability & $40.7 \pm 11.3(19-61)$ \\
AVLT, delayed recall & $7.3 \pm 4.4(0-14)$ \\
TMT, part A (time to complete, s) & $49.8 \pm 25.2(30-123)$ \\
TMT, part B (time to complete, s) & $118.5 \pm 64.2(51-276)$ \\
Stroop color-word test (delay for incongruent vs neutral & $37.3 \pm 17.2(2-68)$ \\
$\quad$ condition, s) & \\
Verbal fluency, phonematic (no. of words) & $13.9 \pm 4.1(7-25)$ \\
Verbal fluency, categories (no. of words) & $20.0 \pm 7.2(6-35)$ \\
Digit span, forward & $6.6 \pm 2.0(3-10)$ \\
Digit span, backward & $5.1 \pm 1.5(2-7)$ \\
Geriatric Concentration Test (AKT, aggregated value) & $52.3 \pm 3.5(43-55)$ \\
\hline
\end{tabular}

Data are given as mean $\pm S D$ and range (minimum to maximum).

TMT, Trail-making test; AKT, Alters-Konzentrations-Test; AVLT, Auditory Verbal Learning Test.

\section{Materials and Methods}

\section{Study approval}

This study was approved by the ethics committee of the Charité University Hospital (Berlin, Germany) and was in accordance with the declaration of Helsinki. All subjects gave informed written consent before participation in the study and received a small reimbursement at the end.

\section{Participants}

Twenty-two patients ( 10 female, mean age 71.2 years \pm 8.79 SD; range: $50-81$ years) with MCI recruited from the memory clinic of the Department of Neurology of the Charité University Hospital participated in the present study. MCI patients (amnestic; single and multiple domain) were diagnosed clinically according to Mayo Clinic criteria based on subjective cognitive complaints and objective memory impairment in standardized tests. These reflected scores of at least 1 SD (i.e., including both early and late MCI; Jessen et al., 2014) below age- and education-specific norm in relevant subtests of the Consortium to establish a Registry for Alzheimer's Disease (CERAD-Plus; www.memoryclinic.ch) test battery (relevant subtests: total word list, delayed recall word list/figures) or the German version of the Auditory Verbal Learning Test (AVLT; Helmstaedter et al., 2001) with relatively preserved general cognition, no impairment in activities of daily living, and no dementia (Sperling et al., 2011).

Exclusion criteria comprised Mini Mental Status Examination scores $<24$ and history of severe untreated medical, neurological, and psychiatric diseases; sleep disorders; alcohol or substance abuse; brain pathologies identified in the MRI scan; intake of medication acting primarily on the central nervous system (e.g., antipsychotics, antidepressants, benzodiazepines, or any type of over-the-counter sleep-inducing drugs such as valerian); and nonfluent German language abilities. Moreover, psychiatric comorbidity was monitored by Beck's Depression Inventory II (BDIII; exclusion if score $\geq 19$; Kühner et al., 2007) and MRI of the brain was performed to exclude major brain pathologies such as brain tumor and previous stroke. Further, participants were excluded from the analysis if they failed to sleep long and deep enough for at least three so-tDCS/sham blocks, which corresponded to $\sim 22 \mathrm{~min}$ spent in sleep stage 2 or slowwave sleep. This is due to our stimulation protocol, which was designed to prevent application of so-tDCS during inappropriate brain states (wake, sleep stage 1, or REM sleep).

In total, data from 6 patients had to be excluded due to insufficient sleep $(n=2)$, inability to complete computerized tasks $(n=3)$, and a previously undetected psychiatric disorder $(n=1)$, resulting in a final group of 16 patients who completed the experiment. Excluded patients did not differ in baseline parameters from this cohort apart from education duration $(p=0.048$, all others $p>0.2$; see Table 1 for baseline characteristics), with higher mean education duration for excluded participants due to an outlier (30 years). 


\section{Baseline assessments}

A comprehensive neuropsychological testing for assessment of general cognitive status was administered to each participant comprising memory performance (German version of Auditory Verbal Learning Test, AVLT; Helmstaedter et al., 2001), working memory (digit span; Wechsler, 1997), executive functions (Stroop color-word test; Van der Elst et al., 2006), verbal fluency (Regensburg Verbal Fluency Test; Aschenbrenner et al., 2000), processing speed and set shifting (Trail Making test, part A and B; Tombaugh, 2004), and selective attention and concentration (Gatterer, 2008). Furthermore, the neuropsychology battery developed by the CERAD-Plus was administered. The affective state at the time of the testing was assessed using the Positive and Negative Affect Schedule (PANAS; Watson et al., 1988). For baseline characteristics, see Table 1.

In addition, questionnaires regarding recent sleep habits (the German version of Morningness-Eveningness-Questionnaire; Griefahn et al., 2001), sleep quality (Pittsburgh Sleep Quality Index; Buysse et al., 1989), daytime sleepiness (Epworth Sleepiness Scale; Johns, 1991), and the Essen questionnaire on age and sleepiness (Essener Fragebogen Alter-und Schläfrigkeit; Frohnhofen et al., 2010) were administered. Daily sleep diaries and actigraphy (GT3X; ActiGraph) were applied to monitor habitual bedtimes and wake times $7 d$ before the experimental nap sessions. These data verified that participants adhered to a regular sleep schedule.

\section{Study design}

The experimental procedure was identical to Ladenbauer et al. (2016). In brief, patients were tested in a balanced crossover design in a stimulation and sham condition ( $n=8$ participants received so-tDCS and $n=8$ received sham on the first experimental nap) that were separated by an interval of at least 2 weeks to prevent carryover effects. Before experimental nap sessions, participants underwent an adaptation nap in the laboratory.

All nap sessions took place at the sleep laboratory of the Free University Berlin, Germany. Upon arriving at 11:30 A.M., participants were prepared for EEG recordings and then tested on two declarative memory tasks (a verbal paired-associate learning and visuospatial learning including picture and location memory), followed by a procedural memory task (finger sequence tapping). At 2:00 P.M., after a standardized small meal and preparation for so-tDCS, participants were asked to attempt to sleep for a period of $90 \mathrm{~min}$, followed by memory tests $30 \mathrm{~min}$ after awakening.

Before learning and prior to retrieval after the nap, attentional capabilities (Test of Attentional Performance; Zimmermann and Fimm, 1995), the affective state (PANAS scales; Watson et al., 1988), sleepiness (Tiredness Symptoms Scale; Bes et al., 1992; Visual Analog Scale, VAS; Luria, 1975) and activation (VAS for tension) were assessed to control for possible confounding effects. For a schematic representation of the experimental procedure, see Figure $1 a$.

\section{so-tDCS}

The stimulation protocol was identical to that described previously by Ladenbauer et al. (2016). Stimulation electrodes (8 mm diameter) were positioned bilaterally at frontal locations F3 and F4 of the international 10-20 system (mounted into an EASY cap; Falk Minow Services), with reference electrodes placed at each mastoid (ipsilateral; likewise $8 \mathrm{~mm}$ diameter). Anodal current was applied by a battery-driven stimulator (DC-Stimulator; NeuroConn; current split to bilateral electrode sites) and oscillated sinusoidally at a frequency of $0.75 \mathrm{~Hz}$ (between 0 and 262.5 $\mu \mathrm{A})$, resulting in a maximum current density of $0.522 \mathrm{~mA} / \mathrm{cm}^{2}$. Electrode resistance was $<5 \mathrm{k} \Omega$.

so-tDCS started 4 min after the subject had entered stable non-rapid eye movement (NREM) sleep stage 2 and was delivered in a blockwise manner, each as a 5 min block of stimulation separated by stimulationfree interblock intervals of (at least) $1 \mathrm{~min} 40 \mathrm{~s}$. The marker for the beginning of each $1 \mathrm{~min}$ stimulation-free interval (for analyses of immediate stimulation induced effects) was always set manually $40 \mathrm{~s}$ after the end of each stimulation period to exclude the strong and long-lasting stimulation-induced drifts visible in our unfiltered online EEG signal from the analysis (interval of analysis will be referred to hereafter as " 1 min stimulation-free interval"). As described in Ladenbauer et al. (2016), the number of 5 min stimulation blocks ( $3-5$ blocks; 3 were required for inclusion) and the duration of the intermediate stimulation-free intervals depended on the individual subject's sleep, as sleep was monitored after each stimulation block and each stimulation block was only initiated during NREM sleep stage 2, 3, or 4 . We prolonged a stimulation-free interval if the subject moved from sleep stages 2, 3, or 4 to sleep stage 1 , REM sleep, or even wakefulness after an so-tDCS/sham block, with the aim of selectively stimulating in deeper sleep stages (protocol adopted from Antonenko et al., 2013). During each stimulation-free interval, online sleep scoring was performed and the next stimulation was delayed until the subject had reentered sleep stage 2 for $1 \mathrm{~min}$. Because previous studies indicated that so-tDCS effects critically depend on ongoing brain state (Kirov et al., 2009; Marshall et al., 2011), this protocol was chosen to account for higher sleep fragmentation in older adults to prevent application of so-tDCS during inappropriate brain states such as wake, sleep stage 1 , or REM phases.

During the sham session, stimulation electrodes were placed identical to the so-tDCS session, but the tDCS device remained off. The same criteria as for the so-tDCS condition were applied for the sham condition (first sham block began 4 min after onset of sleep stage 2; for subsequent sham blocks, sleep stage 2 or slow-wave sleep was required). Likewise, the first $40 \mathrm{~s}$ after each sham block were excluded from analyses. Participants were blinded for stimulation condition throughout the study. After completing all study-related procedures, they were asked whether they were able to guess in which experimental nap the stimulation had been applied and whether they felt any sensations during the naps.

\section{Sleep monitoring and preprocessing}

During naps EEG was recorded from 26 scalp sites (FP1, FP2, AFz, F7, Fz, F8, FC5, FC1, FC2, FC6, C3, Cz, C4, T7, T8, CP5, CP1, CP2, CP6, P7, P3, $\mathrm{Pz}, \mathrm{P} 4, \mathrm{P} 8, \mathrm{O} 1$, and $\mathrm{O} 2$ ) using $\mathrm{Ag}-\mathrm{AgCl}$ ring electrodes placed according to the extended 10-20 international EEG system. FCz was used as ground site. Data were recorded with the BrainAmp amplifier system (Brain Products) at a sample rate of $500 \mathrm{~Hz}$ and band-pass filtered between 0.05 and $127 \mathrm{~Hz}$. All electrode recordings were referenced to an electrode attached to the nose. Impedances were $<5 \mathrm{k} \Omega$. In addition, EMGs at the chin as well as horizontal and vertical EOGs were recorded according to standard sleep monitoring.

Following the application of a notch filter (centered at $50 \mathrm{~Hz}$ with a bandwidth of $5 \mathrm{~Hz}$ ), a combined semiautomated and visual rejection of raw data was applied to eliminate epochs contaminated by artifacts. This processing was done with BrainVision Analyzer software (version. 2.0; RRID:SCR_002356; Brain Products).

\section{EEG analyses}

We performed spectral and phase amplitude coupling analyses for the 1 min stimulation-free intervals after each so-tDCS and sham block. Depending on the number of actually performed stimulations or sham stimulations in a subject, three to five intervals were used for the analysis.

Spectral power. Spectral power was calculated for each $1 \mathrm{~min}$ stimulation-free interval per electrode using the fast Fourier transform on up to 11 overlapping (by $5 \mathrm{~s}$ ) artifact-free segments each lasting $10 \mathrm{~s}$. Corresponding intervals were used for the sham session. On each of these $10 \mathrm{~s}$ segments of EEG data, a Hanning window was applied before calculating the power spectra (frequency resolution $0.06 \mathrm{~Hz}$ ). Subsequently, mean power $\left(\mu \mathrm{V}^{2}\right)$ was calculated over the frequency bands of interest: SO $(0.5-1 \mathrm{~Hz})$ and fast spindles $(12-15 \mathrm{~Hz})$. We additionally considered the slow spindle frequency band slow spindle $(8-12 \mathrm{~Hz})$ to compare with previous results (Marshall et al., 2006). Topographic regions of interest (ROIs) for these frequency bands were selected according to previous research (Mölle et al., 2011; Ladenbauer et al., 2016). The electrode sites $\mathrm{FC} 1, \mathrm{Fz}$, and $\mathrm{FC} 2$ and $\mathrm{CP} 1, \mathrm{Cz}$, and $\mathrm{CP} 2$ were pooled into two ROIs, frontal and centroparietal, respectively. Thus, mean spectral power reflects an average over the electrodes of each ROI. BrainVision Analyzer software was used to perform spectral power analyses.

Phase-amplitude coupling (PAC). To assess the temporal relationships between memory-relevant oscillations in the EEG signal, we used an event-locked analysis based on Staresina et al. (2015) using the FieldTrip 
toolbox (RRID:SCR_004849; Oostenveld et al., 2011) for MATLAB (The MathWorks, RRID:SCR_001622) as well as custom MATLAB functions. Specifically, we characterized SO-to-spindle PAC for the frontal ROI (Fz, $\mathrm{FC} 1$, and FC2) and the centroparietal ROI (Cz, CP1, and CP2) by applying the following procedures:

(i) SO events were identified for each subject, condition, and electrode based on an established detection algorithm (Mölle et al., 2011, 2002). First, EEG data were filtered between 0.16 and $1.25 \mathrm{~Hz}$ (two-pass FIR band-pass filter, order $=3$ cycles of the low cutoff frequency). Only artifact-free data were used and periods of wake state and REM sleep were also excluded for event detection. Second, periods of SO candidates were determined as the time between two successive positive-to-negative zero crossings in the filtered signal. For the next step, events that met the SO duration criteria (period $\geq 0.8 \mathrm{~s}$ and $\leq 2 \mathrm{~s}$, corresponding to $0.5-1.25 \mathrm{~Hz}$ ) were selected. Third, event amplitudes were determined for the remaining SO candidates (trough-to-peak amplitude between two positive-tonegative zero crossing). Events that also met the SO amplitude criteria ( $\geq 75 \%$ percentile of SO candidate amplitudes; i.e., the $25 \%$ of events with the largest amplitudes) were considered SO events. Finally, artifactfree epochs $(-2.5$ to $+2.5 \mathrm{~s})$ time locked to the SO down-state in the filtered signal were extracted from the unfiltered raw signal for all events.

(ii) Time-frequency representations (TFRs) were calculated per event epoch and channel for frequencies $5-20 \mathrm{~Hz}$ in steps of $0.25 \mathrm{~Hz}$ using a sliding (10 ms steps) Hanning tapered window with a variable, frequencydependent length (mtmconvol function of the FieldTrip toolbox). The window length always comprised a full number of five cycles to ensure reliable power estimates. TFRs were then normalized as difference to pre-event baseline ( -2.5 to $-1.2 \mathrm{~s}$ of the epoch) and averaged per subject, condition, and ROI.

(iii) To quantify synchronization and locking between the (modulating) SO and the (modulated) oscillatory fluctuations of the fast spindle power, we calculated the phase values of these time series and applied a synchronization index. Phase values were calculated for all time points of each extracted $\mathrm{SO}$ event and the corresponding fast spindle power fluctuation using the Hilbert transform. Spindle power fluctuation time series were obtained by the TFR bins averaged across the respective frequencies and up-sampled to the sampling frequency of $500 \mathrm{~Hz}$. To ensure proper phase estimation, both SO and spindle power fluctuation time series were filtered beforehand in the range of the modulating SO event (0.5-1.25 Hz; two-pass FIR band-pass filter, order $=3$ cycles of the low cutoff frequency). The synchronization index (SI) was then calculated between the two phase value time series for each event epoch and electrode. The resulting SI is a complex number of which the radius $(r)$ indicates the strength of locking between the modulating SO and the modulated oscillatory fast spindle power fluctuation, and the angle $(\varphi)$ represents the phase shift between these oscillations. In other words, $\varphi$ indicates the phase of the $\mathrm{SO}$ at which fast spindle power is maximal across time. It was obtained by the following:

$$
\mathrm{SI}=\frac{1}{m} \sum_{j=1}^{m} \mathrm{e}^{\mathrm{i}\left[\theta_{\mathrm{SO}}\left(\mathrm{t}_{\mathrm{j}}\right)-\theta_{\mathrm{sp}}\left(\mathrm{t}_{j}\right)\right]}
$$

where $m$ is the number of time points, $\theta_{\mathrm{SO}}\left(\mathrm{t}_{\mathrm{j}}\right)$ is the phase value of the SO time series at time point $t_{j}$, and $\theta_{s p}\left(t_{j}\right)$ is the phase value of the fluctuations of the fast spindle power time series at time point $t_{j}$ (Cohen, 2008). The interval for computing the SI was $-1 \mathrm{~s}$ to $+1 \mathrm{~s}$ around the $\mathrm{SO}$ center. The distribution of SIs per condition and ROI are visualized in Figure $3 d$. For statistical analyses (see below), SIs were averaged per subject, condition, and ROI.

Sleep architecture. The sleep structure, including the time and proportion spent in different sleep stages, was determined based on polysomnographic criteria according to Rechtschaffen and Kales (1968). For this purpose, EEG data were down-sampled to $250 \mathrm{~Hz}$ and $30 \mathrm{~s}$ epochs were scored manually by means of Schlafaus software (Steffen Gais) in sleep stages 1,2,3, and 4 and REM sleep, epochs of wakefulness, or movement artifacts. Epochs during so-tDCS were not scored due to strong artifacts from so-tDCS in the EEG signal. Likewise, corresponding epochs in the sham session were not scored to obtain comparable time and proportions of sleep stages. Scoring for the $1 \mathrm{~min}$ stimulation-free intervals (between the 5 min blocks of acute stimulation) was additionally performed for $10 \mathrm{~s}$ epochs.

\section{Memory tasks}

All memory tasks were performed with Presentation software (version 14.8; RRID:SCR_002521; Neurobehavioral Systems) and parallel versions were used for all tasks in the two experimental nap sessions. All memory tasks were also previously administered in Ladenbauer et al. (2016). In the visuospatial and verbal memory tasks, subjects were instructed to memorize items for a later recall, but no specific strategy was recommended. There was no overlap regarding the stimuli used in the visuospatial and the verbal memory tasks.

We focused on visual recognition memory as the primary behavioral measure because it is affected early in the course of $\mathrm{AD}$ (Barbeau et al., 2004; Wolk et al., 2008; Besson et al., 2015), yes/no recognition performance reliably allowed to predict group membership (AD, MCI, healthy control; Bennett et al., 2006), and recognition in contrast to recall paradigms permits examination of all items and not just explicitly recalled ones. For comparison with previous studies, we additionally assessed location recall (Prehn-Kristensen et al., 2014; Del Felice et al., 2015) and associative word pair recall (Marshall et al., 2006; Eggert et al., 2013; Prehn-Kristensen et al., 2014; Westerberg et al., 2015).

Visuospatial memory task. The visuospatial memory task consisted of 38 neutral pictures (objects, plants, scenes, and landscapes taken from the International Affective Picture System (Lang et al., 1999; MULTIMOST, Schneider et al., 2008) that appeared randomly at 1 of the 4 possible quadrants on the screen for $2 \mathrm{~s}$ with an interstimulus interval of $1 \mathrm{~s}$ (see Fig. 1b). To account for primacy and recency effects, four additional pictures (two at the beginning and two at the end) were included and disregarded in the analyses. Participants were instructed to memorize both the pictures (picture memory) and their locations (location memory). During recognition testing (before and after sleep), each picture (38 studied and 38 new pictures in random order) was displayed in the center of the screen $(3 \mathrm{~s})$, while participants indicated by button press whether they recognized each picture (yes for "old" decision and no for "new" decision). If participants recognized an item ("old" decision), then they were also required to indicate in which quadrant they believed the picture was presented during acquisition.

"Old/new" decisions in this task resulted in four possible response categories: hits (correct "old" judgements), correct rejections (correct "new" judgements), false alarms (incorrect "old” judgments), and misses (incorrect "new" judgements). As a measure for picture recognition memory accuracy, the percentage of correct responses was calculated as follows: proportion of hits + proportion of correct rejections. Further, potential response biases were considered by calculating the sum of the proportion of hits and false alarms.

For the accuracy of location memory, both correctly and incorrectly retrieved picture locations were taken into account and calculated as follows: number of correctly retrieved locations/number of hits - number of falsely retrieved locations/number of hits. Data from one participant was excluded from analyses for this task due to technical problems.

Given the high proportion of complex picture stimuli (scenes and landscapes) and the similarity of targets and foils, picture recognition performance here comprised recollection as well as familiarity processes (Mandler, 2008; Migo et al., 2009), both of which have been shown to be affected in MCI and are thus of clinical relevance (Dudas et al., 2005; Bennett et al., 2006; Algarabel et al., 2012; Wolk et al., 2008, 2013).

Verbal memory task. Participants viewed 40 semantically related German word pairs (category-instance pairs: e.g., fruit-banana) that appeared centrally on the screen for $5 \mathrm{~s}$ with an interstimulus interval of $100 \mathrm{~ms}$. Additional four word pairs were presented (two at the beginning and two at the end) to prevent primacy-recency effects. Following encoding, an initial cued-recall test was performed. The category name (cue) appeared centrally and participants were instructed to say the respective stimulus (instance) word aloud after indicating (by button press within $10 \mathrm{~s}$ ) whether they remembered the respective instance word. Subsequently, the correct word pair was displayed for $2.5 \mathrm{~s}$. This initial cued-recall test provided an additional learning opportunity to help reach the $\sim 60 \%$ correct responses at the subsequent cued recall test. The 
immediate cued-recall and delayed cued-recall test (after sleep) were administered without additional presentation of the correct word pairs. Word pairs were presented in a different randomized order to prevent serial learning in each encoding and recall test. Participants' verbal responses were recorded and cued recall performance was obtained by the proportion of the correct retrieved targets in the immediate and delayed recall test, respectively.

Moreover, we tested the impact of stimulation on interference rate (proportion of incorrect instance words in a nap session that were correct responses to the same categories in a previous session). Data from one participant were excluded from analyses for this task because she failed to follow task instructions.

Please note that our verbal task differed from the tasks used in Marshall et al. (2006) and Westerberg et al. (2015) in that our word pair lists were designed to achieve high comparability between word pairs for the two experimental naps and to exclude emotional words (for more details, see Ladenbauer et al., 2016). Although comparability between word pair lists was increased by the category-instance (e.g., fruit-banana) structure, the word pairs exhibited stronger semantic associations compared with previous studies [Westerberg et al., 2015: mean cue-to-target association strength of 0.04 (applicable to $31 \%$ of the pairs) compared with 0.08 in our verbal task (applicable to $29 \%$ of the pairs); see Nelson et al., 2004].

Procedural task. A sequential finger tapping task adapted from Walker et al. (2002) was used to investigate so-tDCS effects on procedural memory. Participants were asked to repeatedly tap a five-digit sequence (e.g., 4-2-3-1-4) that was displayed on the screen with the nondominant left hand as accurately and as quickly as possible within a $30 \mathrm{~s}$ interval (trial). During pre-nap testing (learning), participants performed on 12 trails separated by 30 s breaks. Testing after nap (retrieval) contained four trials with breaks. Performance at learning and retrieval testing was determined by averaged correctly tapped sequences during the final three trials, respectively. Four participants failed to complete this task and were therefore excluded from analyses of this task.

\section{Statistical analyses}

Spectral power. Differences between the two stimulation conditions (so-tDCS and sham) in the five outcome measures frontal and centroparietal SO and fast spindle power as well as frontal slow spindle power were evaluated using a linear mixed model with random intercepts (LMM) (Verbeke and Molenberghs, 2000). This model was chosen to account for characteristic individual differences in sleep physiology (Buckelmüller et al., 2006; Tucker et al., 2007) and for unequal numbers of spectral power data points across subjects and condition (three to five applied so-tDCS and sham blocks depending on the individual subject's sleep).These inequalities arose from the protocol chosen to prevent application of sotDCS during inappropriate brain states.

In brief, the model is described by the following:

$$
y_{i j}=\beta_{0}+\sum_{k=1}^{6} \beta_{k} x_{k i j}+z_{j}+\epsilon_{i j}
$$

where $y_{i j}$ denotes the log-transformed spectral power value of participant $j \in\{1,2, \ldots, 16\}$ in interval $i \in\{1,2,3,4,5\}, \beta_{0}$ is the fixed intercept and $\beta_{1}, \ldots, \beta_{6}$ are the regression coefficients that correspond to the independent variables for stimulation, $x_{1 i j} \in\{0,1\}$, baseline, $x_{2 i j}$ (see below), (centered) time, $x_{3 i j}=i-3$, (centered) time ${ }^{2}, x_{4 i j}=(i-3)^{2}$, time $\times$ stimulation, $x_{5 i j}=(i-3) x_{2 i j}$, and time ${ }^{2} \times$ stimulation, $x_{6 i j}=(i-3)^{2} x_{2 i j}$. The subject-specific random intercept is given by $z_{j}$ and $\epsilon_{i j}$ denotes the residual (or random error); that is, the five time points ( $1 \mathrm{~min}$ stimulation-free interval after each stimulation block) were level-one units nested in subjects (level-two units). The model assumed that slopes were similar (no random slope model) since there was no previous evidence on interindividual differences in stimulation effects (slopes) on sleep physiology. The baseline variable $\left(x_{2 i j}\right)$ as covariate served to adjust for baseline differences in each frequency band, respectively. This variable took the subject-specific log-transformed spectral power value calculated from a $1 \mathrm{~min}$ interval preceding the first stimulation/sham block. As spectral power data did not exhibit a normal distribution, they were $\log$ transformed before application of the LMM. Note that the values of the variables for stimulation and baseline did not vary across intervals. The squared centered time variable $\left(x_{4 i j}\right)$ was incorporated to test for curvilinear course of $\mathrm{SO}$ and spindle power over intervals, as observed in healthy older adults (Ladenbauer et al., 2016). By the interaction time $\times$ stimulation, we assessed whether the slopes of the curves differed between the stimulation conditions and an interaction term time ${ }^{2} \times$ stimulation was included to test whether the shape of the curves differed between the stimulation conditions.

PAC. We tested for significant event-locked power changes in the TFR due to stimulation using a two-tailed paired-samples $t$ test (group-level statistics). To correct for multiple comparisons $(-1.2 \mathrm{~s}$ to $+1.2 \mathrm{~s} \times 5-20$ $\mathrm{Hz}$ ), a cluster-based permutation procedure was applied as implemented in FieldTrip (Maris and Oostenveld, 2007; Staresina et al., 2015). The initial threshold for cluster definition was set to $p<0.025$ and the final threshold for significance of the summed $t$ value within clusters was set to $p<0.05$.

To assess whether the (circular) SI angles were distributed nonuniformly with a specified mean, we applied the V test (Berens, 2009). Using this test, the alternative hypothesis $\mathrm{H} 1$ states that the population is clustered around a known mean direction. In the current case, we expected maximal fast spindle power around the SO up-state; that is, clustering around 0 deg (Staresina et al., 2015).

Furthermore, we calculated the resultant vector length which indicates the degree of concentration of SI angles (Berens, 2009). For a particular condition and ROI it is calculated by the following:

$$
\left|\frac{1}{n} \sum_{j=1}^{n} e^{i \varphi_{j}}\right|
$$

where $\varphi_{j}$ is the angle of the SI for subject $j \in\{1, \ldots, n\}$.

Sleep architecture. To evaluate so-tDCS effect on sleep structure, $t$ tests (or Wilcoxon signed-ranks tests if indicated) were performed for the proportion of time spent in different sleep stages (in \%) during the entire nap and during the 1 min stimulation-free intervals.

Memory tasks. so-tDCS effects on retention performances in the memory tasks were tested by repeated-measures ANOVA (rmANOVA), including the within-subject factors stimulation (so-tDCS vs sham stimulation) and time point (pre-nap vs post-nap). The GreenhouseGeisser correction of degrees of freedom was applied when appropriate.

Statistical analyses for memory tasks, power measures, and sleep structure were performed using SPSS (version 22.0; RRID:SCR_002865; IBM); PAC behavior was statistically analyzed using the FieldTrip (Oostenveld et al., 2011) and CircStat (Berens, 2009) MATLAB toolboxes as well as custom MATLAB functions. A two-sided significance level $\alpha$ was set to 0.05 in all analyses. Given multiple testing for the primary parameters of interest $[n=5$; visual recognition performance (percentage correct score) and the four memory-related EEG spectral power measures, i.e., frontal and centroparietal SO and fast spindle power], we applied the Benjamini-Hochberg correction (Benjamini and Hochberg, 1995) to control for the false discovery rate at $q=0.05$. The corrected significance level is denoted as $q^{\star}$ in the Results section. To correct for multiple comparisons in the statistical TFR analysis, a cluster-based permutation method was applied (see above). Reported effect size for behavioral effects is partial $\eta^{2}$ (subsequently shortened to $\eta^{2}$ ). In addition, we calculated model-based standardized effect sizes for the LMM regarding stimulation effects on EEG power (Cohen's $d$ ) using marginal mean differences and SD based on SEs (Hedges, 2007). All other tests and comparisons were related to secondary hypotheses and $p$-values should be interpreted in a framework of exploratory analysis.

\section{Results}

We analyzed EEG data and memory performance of $16 \mathrm{MCI}$ patients ( 7 female, mean age 70.6 years $\pm 8.9 \mathrm{SD}$ ) who were tested on memory tasks before and after a 90 min nap with either so-tDCS or sham stimulation (two sessions, balanced crossover design). A schematic diagram of the experiment is shown in Figure 1. We focused on visual recognition memory, whose im- 
a

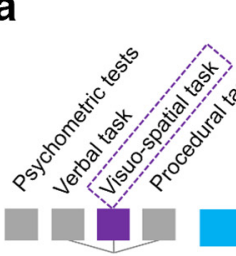

Encoding

\& Recall
Wake
REM
S

Wake
REM
S1
S2

S3

S4

-min stimulation-free intervals for analyses

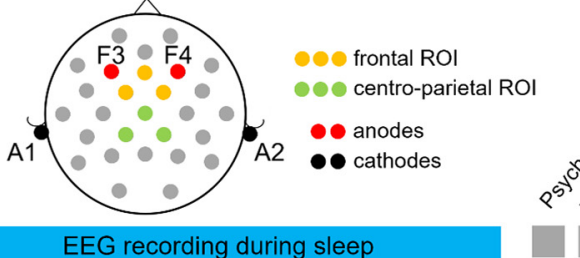

EEG recording during sleep
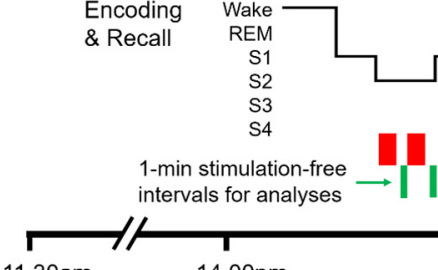

$14.00 \mathrm{pm}$
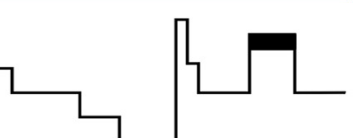

1

5 -min blocks of so-tDC
starting after $\mathrm{S} 2$ onset $\leftarrow 5$-min blocks of so-tDCS/sham

11.30am

$15.30 \mathrm{pm}$

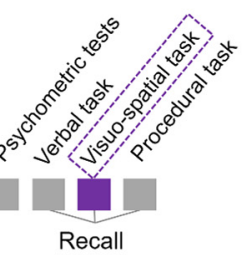

b

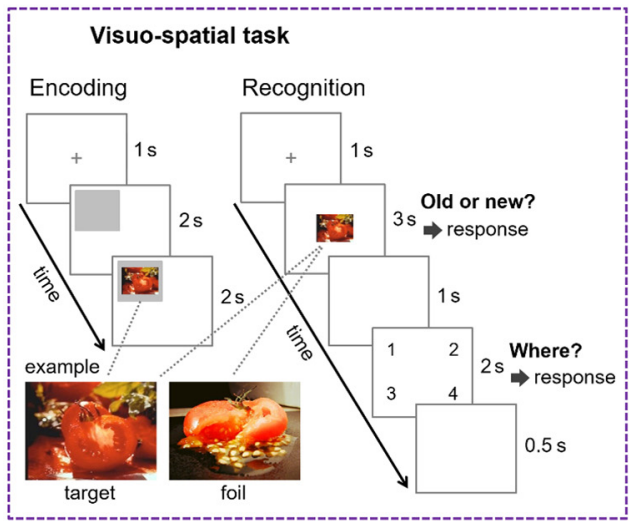

Figure 1. Study design. $\boldsymbol{a}$, Subjects learned a verbal, a visuospatial, and a procedural task in the indicated order after psychometric control tests. During a subsequent 90 min nap (2:00 P.M. to $3: 30$ P.M.), EEG was recorded and either slow oscillatory transcranial direct current stimulation (so-tDCS) or sham stimulation was applied (within-subject design, randomized order) in up to five 5 min blocks that started after sleep stage 2 onset. According to the example hypnogram stimulation, blocks are discontinued as the subject moves from sleep stage 2 to stage 1 , and resumed after the subject again enters sleep stage 2 (or lower). Thick line in the hypnogram is REM. S1-S4, Sleep stages 1-4. Recordings from electrodes Fz, FC1, and FC2 (frontal ROI), and CZ, CP1, and CP2 (centroparietal ROI) during 1 min stimulation-free intervals starting $40 \mathrm{~s}$ after each so-tDCS/sham block were used for spectral and phase amplitude coupling analyses. Memory retrieval and psychometric control tests were performed $30 \mathrm{~min}$ after the nap. $\boldsymbol{b}$, Example encoding and recognition trials of the visuospatial memory task. For encoding, a gray rectangle was presented at one of the quadrants of the screen after a fixation cross and followed by a neutral picture within the gray region for $2 \mathrm{~s}$. For recognition, a picture was displayed in the center of the screen for $3 \mathrm{~s}$ after a fixation cross. Within this time period, subjects were asked to indicate whether they believed they had seen the picture earlier. If subjects recognized an item, then they also indicated in which quadrant they believed the item had been presented.

pairment occurs early in the course of AD (Barbeau et al., 2004) and thus constitutes a sensitive target for interventional approaches in MCI (for details, see Materials and Methods). For comparison with previous studies, we additionally assessed word pair recall.

We first asked whether so-tDCS beneficially affects memoryrelevant sleep measures. For this purpose, we focused on the immediate effects on $\mathrm{SO}$ and fast spindles measured by EEG during 1 min lasting stimulation-free intervals after up to 5 so-tDCS/ sham blocks. We assessed the power of these oscillations as well as their functional coupling. Based on previous studies, we performed these analyses for frontal and centroparietal ROIs separately (Mölle et al., 2011; Ladenbauer et al., 2016). In addition, we assessed frontal slow spindle power and quantified changes of sleep architecture. We then examined how stimulation changed declarative memory performance and finally explored the relationship between changes of performance and sleep measures.

The number of so-tDCS/sham blocks could vary between sessions as we accounted for the individual subject's sleep by ensuring sleep stage 2 or deeper before every stimulation block (state-dependent stimulation, see Materials and Methods). No significant differences were seen between conditions regarding the number of so-tDCS/sham blocks that participants received (see Table 2, Wilcoxon signed-rank test: $p=0.453$ ). The stimulation was well tolerated by all patients. They did not report any sensation except one patient who indicated a tingling perception. Postexperimental debriefing indicated that most subjects were not able to guess in which nap session so-tDCS was applied $(n=$ 14 answered "do not know" and two subjects correctly guessed the so-tDCS nap). Considering sleep schedules and sleep duration before the experiments, no differences between stimulation conditions were evident (all $p>0.2$ ).

\section{Effects on memory-relevant sleep measures}

Spectral power

To analyze the effects of stimulation on spectral power memoryrelevant frequency bands, we used an LMM, which accounts for individual differences in sleep physiology and unequal numbers of observations across subjects (3-5 so-tDCS/sham blocks, see Materials and Methods). LMM analyses revealed a significant so-tDCS-related enhancement in frontal and centroparietal SO $(0.5-1 \mathrm{~Hz})$ power during the nap [stimulation, mean difference, frontal: $0.28,95 \%$ confidence interval (CI): $0.16-0.40, d=1.16$, $p<0.001, q^{1}=0.01$; centroparietal: $0.19,95 \%$ CI: $0.06-0.31$, $d=0.70, p=0.006, q^{3}=0.03$; Fig. 2). This enhancement could not be explained by the baseline SO power (during 1 min before the first so-tDCS/sham block) for which no significant difference between so-tDCS and sham condition was observed (frontal: $t_{(15)}=$ $1.65, p=0.121$; centroparietal: $\left.t_{(15)}=1.20 p=0.249\right)$. Furthermore, baseline $\mathrm{SO}$ power was not significantly associated with $\mathrm{SO}$ activity after the stimulation blocks (baseline, frontal: $\beta_{2}=$ $-0.082, \mathrm{SE}=0.168, p=0.628$; centroparietal: $\beta_{2}=-0.145$, $\mathrm{SE}=0.206, p=0.483)$. With regard to the course of $\mathrm{SO}$ power over $1 \mathrm{~min}$ stimulation-free intervals across the nap independent of condition, we did not observe a significant linear change (time, frontal: $\beta_{3}=0.027, \mathrm{SE}=0.029, p=0.353$; centroparietal: $\beta_{3}=$ $0.027, \mathrm{SE}=0.033, p=0.408)$, but an inverted $\mathrm{U}$-shaped relationship, which was stronger over centroparietal $\left(\right.$ time $^{2}, \beta_{4}=$ $-0.061, \mathrm{SE}=0.027, p=0.025)$ than frontal sites $\left(\mathrm{time}^{2}, \beta_{4}=\right.$ $-0.040, \mathrm{SE}=0.024, p=0.092$ ). Neither the slope nor the curvedness of SO power over intervals differed significantly between conditions (time $\times$ stimulation and time ${ }^{2} \times$ stimulation interactions: all $p>0.2$ ).

This enhancing so-tDCS effect was not restricted to the SO band. For fast spindle power $(12-15 \mathrm{~Hz}), \mathrm{LMM}$ analyses also showed significant stimulation effects (stimulation, mean difference, frontal: $0.14,95 \%$ CI: $0.07-0.21, d=1.02, p<0.001 ; q^{2}=$ 0.02 ; centroparietal: $0.07,95 \%$ CI: $0.003-0.14, d=0.50, p=$ $\left.0.041, q^{5}=0.05\right)$ indicating increased power after so-tDCS compared with sham. These so-tDCS-induced power increases could also not be explained by fast spindle power during baseline because there was no significant difference between so-tDCS and sham during baseline (frontal: $t_{(15)}=1.84, p=0.086$; centropa- 


\begin{tabular}{|c|c|c|c|c|c|}
\hline & so-tDCS & & Sham & & \\
\hline \multicolumn{6}{|l|}{ Distribution of stimulation counts during the nap $(n)$} \\
\hline 5 blocks & 12 & & 10 & & \\
\hline 4 blocks & 2 & & 3 & & \\
\hline \multirow[t]{2}{*}{3 blocks } & 2 & & 3 & & \\
\hline & Mean (SD) & MD & Mean (SD) & MD & $p$ \\
\hline Sleep time excluding movement periods and so-tDCS/sham (min) & $48.8(13.2)$ & 49.5 & $51.6(11.6)$ & 56.00 & $0.569^{a}$ \\
\hline \multicolumn{6}{|c|}{ Proportion of total sleep time (including so-tDCS/sham) spent in different sleep stages (\%) } \\
\hline WASO & $14.7(11.9)$ & 11.3 & $20.36(1)$ & 15.1 & 0.218 \\
\hline NREM stage 1 & $15.1(5.7)$ & 11.8 & $16.6(5.1)$ & 14.1 & 0.959 \\
\hline NREM stage 2 & $27.9(6.7)$ & 28.9 & $24.4(7.8)$ & 22.8 & 0.258 \\
\hline NREM stage 3 & $2.7(1.1)$ & 0.3 & $2.7(1.7)$ & 0.0 & $0.504^{a}$ \\
\hline NREM stage 4 & $1.1(1.1)$ & 0.0 & $0.2(0.1)$ & 0.0 & $1.000^{a}$ \\
\hline REM & $2.3(1.3)$ & 0.0 & $3.1(1.3)$ & 0.0 & $0.600^{a}$ \\
\hline \multicolumn{6}{|c|}{ Proportion of time during stimulation-free intervals spent in different sleep stages (\%) } \\
\hline WASO & $7.44(10.5)$ & 0.0 & $14.7(15.0)$ & 11.7 & $0.062^{a}$ \\
\hline NREM stage 1 & $7.5(11.6)$ & 3.3 & $15.8(19.9)$ & 11.7 & $0.091^{\circ}$ \\
\hline NREM stage 2 & $70.3(21.0)$ & 69.5 & $51.5(18.4)$ & 53.3 & 0.021 \\
\hline NREM stage 3 & $12.8(11.9)$ & 10.0 & $10.4(14.2)$ & 4.9 & $0.451^{\circ}$ \\
\hline NREM stage 4 & $2.8(9.5)$ & 0.0 & $3.1(9.5)$ & 0.0 & $1.000^{\circ}$ \\
\hline REM & $0.0(0.0)$ & 0.0 & $2.3(6.3)$ & 0.0 & $0.180^{\circ}$ \\
\hline
\end{tabular}

Sleep time is the elapsed time from sleep onset to the end of nap. Comparisons are based on paired-samples $t$ test unless indicated otherwise. ${ }^{*} p<0.05$.

WASO, Wake after sleep onset; MD, median.

${ }^{a}$ Wilcoxon signed-rank test due to skewed distributions.
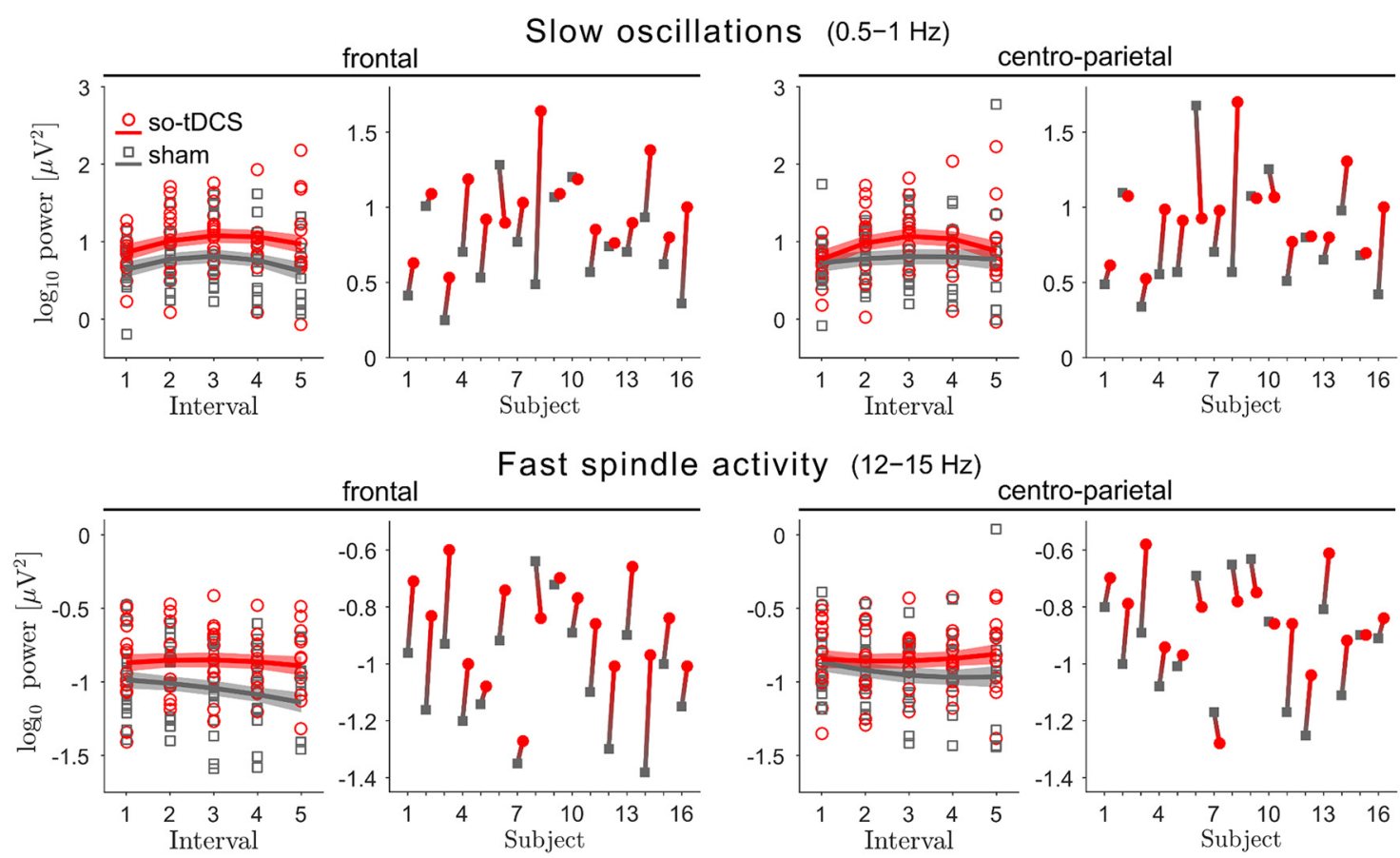
centro-parietal

Figure 2. So-tDCS enhances $\mathrm{EEG}$ power in the $\mathrm{S} 0$ and fast spindle frequency ranges. EEG power in the $\mathrm{SO}(0.5-1 \mathrm{~Hz}$, top) and fast spindle (12-15 Hz, bottom) frequency ranges for the five 1 min stimulation-free intervals, for so-tDCS (red) and sham (gray) condition, and considering the frontal (left) and centroparietal (right) ROI. Mean estimates \pm SEM (shaded regions) from the LMM are included. To the right, individual mean power changes (mean over all intervals for each subject) from sham (gray squares) to so-tDCS (red circles) are indicated for each frequency band and ROI.

rietal: $\left.t_{(15)}=0.88 p=0.393\right)$. Nevertheless, we found that baseline fast spindle power was significantly associated with power in this frequency range during later stimulation free intervals over frontal (baseline, $\beta_{2}=0.463, \mathrm{SE}=0.122, p<0.001$ ) and centroparietal sites (baseline, $\beta_{2}=0.392, \mathrm{SE}=0.146, p=0.010$ ).

We further examined the effect of stimulation on frontal slow spindle power $(8-12 \mathrm{~Hz})$ because a previous study in young subjects found so-tDCS-induced increases in the slow spindle band, but not for fast spindle power (Marshall et al., 2006). In the present study, frontal slow spindle power was significantly increased after so-tDCS compared with sham stimulation (stimulation, mean difference, 0.10 ; 95\% CI: $0.04-0.16, d=0.82, p=$ $0.001)$. Here, baseline frontal spindle power did not significantly differ between so-tDCS and sham condition $\left(t_{(15)}=1.11, p=\right.$ 0.287 ), but, consistent with fast spindle power, baseline slow spindle power was significantly associated with power in this fre- 


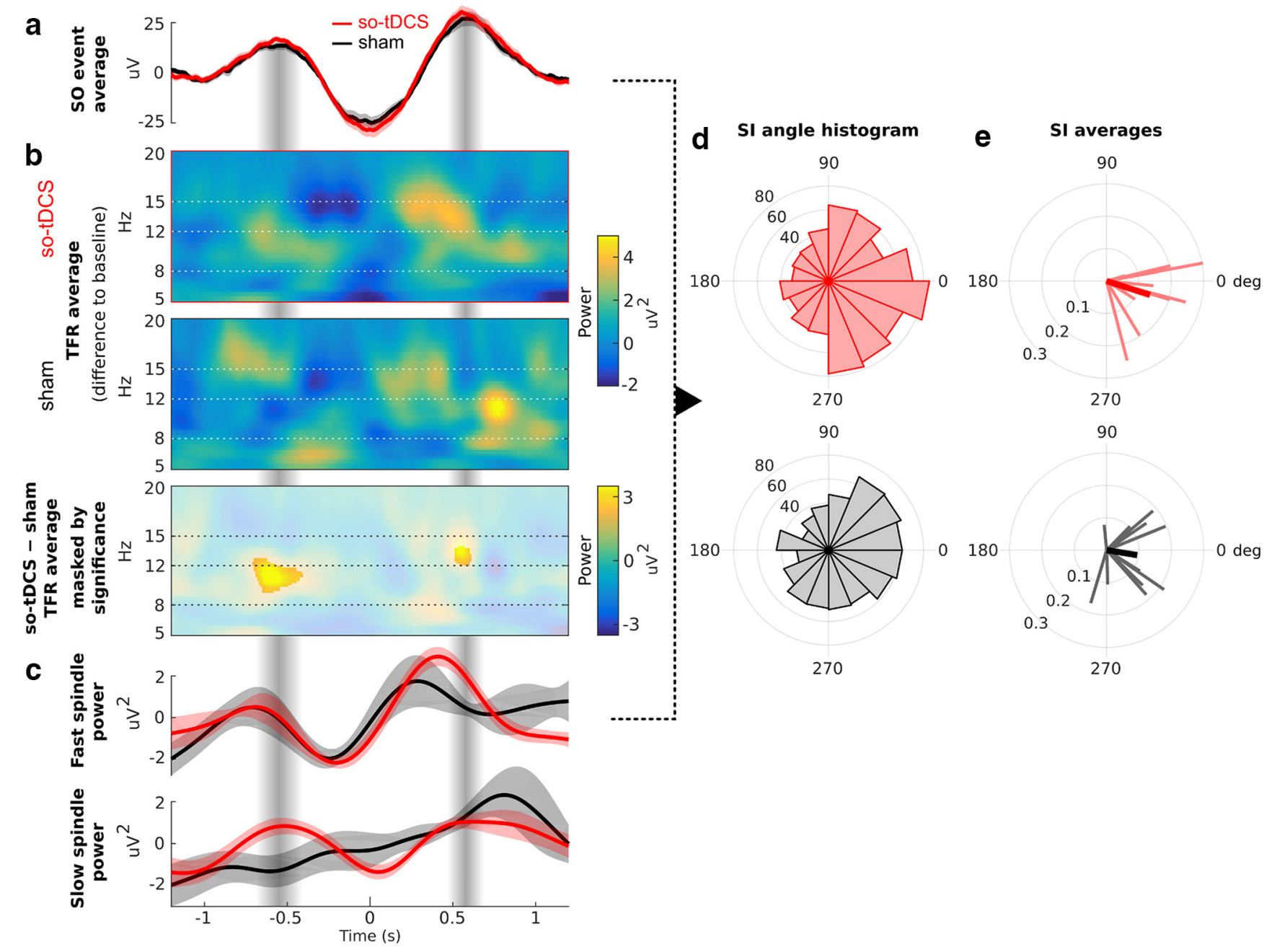

Figure 3. Phase amplitude coupling between $\mathrm{SO}$ and spindle power. $\boldsymbol{a}$, Grand average EEG trace (mean \pm SEM across participants) of a total of 860 events for so-tDCS (red) and 765 events for sham (black) condition aligned to the $\mathrm{SO}$ trough (time 0 ) from the centroparietal ROI and all 1 min stimulation-free intervals. $\boldsymbol{b}$, TFRs locked to the $\mathrm{SO}$ events (from $\boldsymbol{a}$ ) and averaged per condition: so-tDCS (top) and sham (center). Shown are the differences from the pre-event baseline power values $(-2.5 \mathrm{~s}$ to $-1.2 \mathrm{~s})$. Bottom, Difference of these TFRs masked by significance $(p<0.05$, corrected). c, Time course of event-locked average power from the TFRs in $\boldsymbol{b}$ filtered in the range of the modulating $\mathrm{SO}$ for the fast spindle $(12-15 \mathrm{~Hz}$, top) and slow spindle $(8-12 \mathrm{~Hz}$, bottom) frequency ranges (mean \pm SEM across participants). $\boldsymbol{d}$, Histogram of SI angles indicating the phase difference between $S 0$ and the fast spindle power fluctuation (cf. $\boldsymbol{a}$ and $\boldsymbol{c}$ ) for the conditions so-tDCS (top, $n=860$ ) and sham (bottom, $n=765$ ). An angle value of 0 indicates synchrony, whereas 180 deg indicates an anti-phase relationship. A value just below 0 (close to 360 deg) indicates that spindle power tended to peak shortly before the SO peak. Note that the SI angle distribution for so-tDCS indicates that the spindle power peak preferably occurred during the late rising phase of S0. $\boldsymbol{e}$, Sls averaged per subject (thin lines) and across subjects (thick lines) for the two conditions. Note that the angle of the $\mathrm{Sl}$ indicates the phase of $\mathrm{SO}$ at which spindles tend to occur, whereas its radius indicates the strength of locking (coupling) between $\mathrm{SO}$ and the oscillatory spindle power fluctuations.

quency range during stimulation free intervals after so-tDCS/ sham blocks (baseline, $\beta_{2}=0.674, \mathrm{SE}=0.112, p<0.001$ ). No further significant effects for power in the fast and slow spindle frequency ranges were found (all $p>0.1$ ).

$P A C$

To assess functional coupling of SO and spindles, we employed an analytical approach that investigated whether the amplitude (power) of the spindle oscillation is systematically modulated by the phase of the SO (phase-amplitude coupling). Using an eventlocked analysis, we specifically examined the hypothesized nesting of SO and fast spindles. We identified SO events in the EEG according to an established detection algorithm and aligned TFRs of the perievent epochs to the center of the SO trough (see Materials and Methods). SO-to-spindle PAC emerges as power modulation over time in the respective (event-locked) TFR. To ensure reliable PAC results, four patients had to be excluded from this analysis because they exhibited only a very small number of SO events
( $<10$ per electrode). Therefore, $n=12$ were included in the final analysis.

We found that so-tDCS lead to a significant increase in centroparietal fast spindle power during the SO up-phase that follows the event-centering SO trough (down-phase), as well as in centroparietal slow spindle power during the $\mathrm{SO}$ up-phase that precedes the $\mathrm{SO}$ trough (Fig. $3 a-c$ ). It may be noted that the modulation of slow spindle power in the so-tDCS condition led to visibly stronger oscillatory behavior that was phase delayed by $\sim 250 \mathrm{~ms}$ relative to the fast spindle power oscillation (Fig. $3 c$ ). In the frontal ROI we did not observe significant effects of stimulation on PAC assessed in this way.

We further examined this coupling between SO and fast spindles in an event-wise manner using SI, which measures the strength of locking and phase shift between the modulating SO and the modulated oscillatory spindle power fluctuation (see Materials and Methods). The SIs shown in Figure 3, $d$ and $e$, 
emphasize the enhancement of the hypothesized memoryrelevant PAC due to stimulation. The overall distribution of SI angles indicates the phases of $\mathrm{SO}$ at which fast spindles occur preferably. This distribution shows a much stronger peak close to zero (in phase synchrony) for stimulation compared with sham condition (Fig. $3 d$ ) and is more concentrated as measured by the resultant vector length (so-tDCS: 0.20 vs sham: 0.16 ) and a larger proportion of SI angles are in the fourth quadrant (270-360 deg, so-tDCS: $35 \%$ vs sham: $29 \%$ ), indicating that spindle power peak preferably occurred during the late rising phase of SO. This was confirmed by the statistics for the subject-averaged SI angles (Fig. $3 e$ ), for which 8 (so-tDCS) versus 5 (sham) out of 12 are in the fourth quadrant and whose resultant vector length (indicating the degree of concentration across subjects) amounts to 0.89 (sotDCS) versus 0.58 (sham). $V$ test results further demonstrate that, for the stimulation condition, subject-averaged SI angles are substantially tighter concentrated close to 0 (so-tDCS: $\mathrm{V}=10.16$, $p<0.00002$ vs sham: $\mathrm{V}=6.84, p<0.003$ ).

For the frontal ROI, we observed a similar (but weaker) enhancement of SO-to-fast-spindle PAC by stimulation as quantified by $\mathrm{V}$ test statistics for the subject-averaged SI angles (so-tDCS: $\mathrm{V}=8.50, p<0.0003$ vs sham: $\mathrm{V}=5.67, p=0.01$ ) and corresponding resultant vector lengths (so-tDCS: 0.71 vs sham: $0.52)$.

\section{Sleep stages}

Table 2 summarizes total sleep time spent in different sleep stages during the nap and during the 1 min stimulation-free intervals. No significant differences between conditions were found in total sleep time and times spent in the different sleep stages (all $p>$ 0.2 ). However, for the $1 \mathrm{~min}$ stimulation-free intervals, pairwise comparisons yielded a significant difference in NREM sleep stage $2\left(t_{(15)}=2.57, p=0.021\right.$, mean difference $18.86,95 \%$ CI: $3.22-$ 34.51 ). Sleep stage 2 (in \%) was increased after so-tDCS compared with sham, whereas wake time after sleep onset and NREM sleep stage 1 was trendwise reduced (Wilcoxon signed-rank tests: $p=0.062$ and $p=0.091$, respectively).

To summarize, so-tDCS in MCI patients profoundly increased SO power and enhanced power in the fast and slow spindle frequency ranges. PAC analyses further revealed that so-tDCS lead to stronger synchronization between $\mathrm{SO}$ and fast spindle power, in particular by increasing spindle power during late-rising SO up-phases. These beneficial so-tDCS effects were also reflected in the sleep structure during the 1 min stimulationfree intervals, with increased sleep stage 2 and trendwise less sleep stage 1 and wake phases compared with sham condition.

\section{Effects on memory}

To control for potential confounding influences on memory performance, we assessed self-reported mood, activation, sleepiness, and attention before and after the naps. No significant pre- and post-nap differences were found (all $p>0.1$ ). Likewise, we did not find stimulation-dependent changes in attention, mood, and activation (all $p>0.2$ ), but there was a nonsignificant trend toward increased self-reported sleepiness as indexed by the Tiredness Symptoms Scale (physical sleepiness) after naps with so-tDCS $\left(F_{(1,15)}=\right.$ $3.50, p=0.082)$ compared with the sham condition.

We tested whether so-tDCS effects were also reflected in visual memory performance in MCI patients ( $n=15$, see Materials and Methods). Given the (mild) so-tDCS effect on sleepiness, we included sleepiness as covariate in the respective analyses on memory performance (ANCOVA). We found a significant so-tDCS effect on visual memory as assessed by picture recognition accuracy

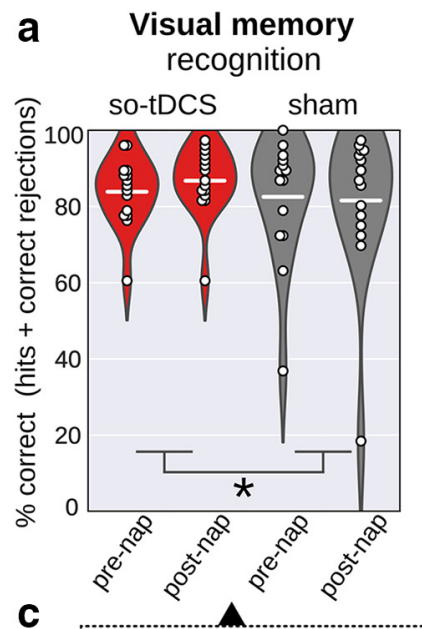

\section{b Verbal memory cued recall}

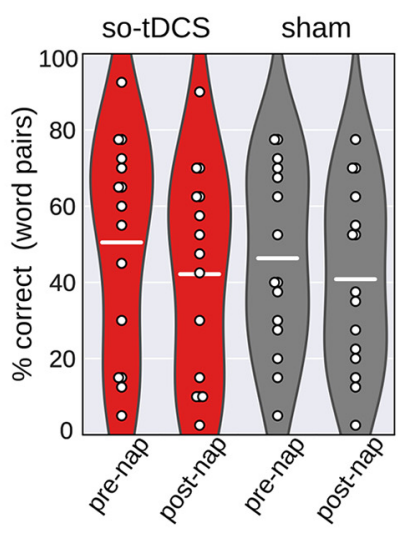

C so-tDCs
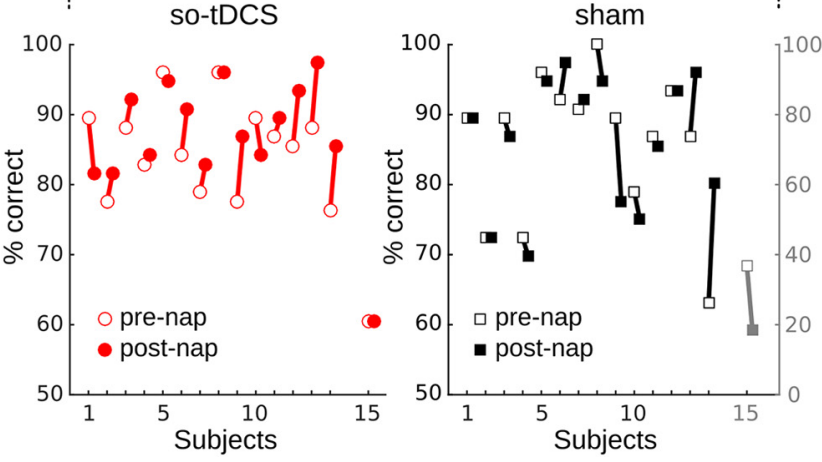

Figure 4. Retention performance in declarative memory tasks in the so-tDCS versus sham condition. $\boldsymbol{a}, \boldsymbol{b}$, Recognition performance (percentage correct: proportion of hits and correct rejections) in the picture memory subtask ( $\boldsymbol{a}$ ) and cued recall performance (percentage correct) in the verbal memory task (b) for so-tDCS (red) and sham condition (gray) measured before (pre-nap) and after (post-nap) the nap. Dots indicate individual performances, a white line represents the mean per condition and time point, violin plots show the distributions across subjects. A significant stimulation effect emerged for picture memory, with higher picture recognition performance after so-tDCS compared with sham condition. ${ }^{*} p<0.05$. c, Picture recognition performance of individuals before and after napping for so-tDCS (left) and sham (right) condition. Note the separate scale for the outlier (subject 15, gray) in sham.

(percentage correct sores: hit rate + correct rejection rate) (stimulation $\times$ time point interaction: $F_{(1,12)}=5.34, p=0.039, q^{4}=$ 0.04; performance change across nap in \%: so-tDCS, 2.89, 95\% CI: 0.24-5.54; sham, $-0.96,95 \%$ CI: $-5.10-3.18$, $\eta^{2}=0.308$; see also Fig. 4 for pre- and post-nap scores). We further tested for pre-nap differences between conditions and possible response bias. Pre-nap visual recognition performance did not differ between sessions $\left(t_{(14)}=0.38, p=0.712\right)$ and no ceiling effect was observable (pre-nap percentage correct: so-tDCS, 83.86, 95\% CI: 78.9-88.8; sham, 82.94, 95\% CI: 73.6-91.5; however, one patient achieved $100 \%$ in the sham condition). With regard to response bias, as indicated by the sum between hit rate and false alarm rate, an impact toward less conservative responding after the nap with so-tDCS compared with sham condition was found $\left(F_{(1,12)}=11.83, p=0.005, \eta^{2}=0.496\right)$. However, examining this effect in more detail by testing so-tDCS effects separately for hit rate and false alarm rate, a significant effect was only apparent for hit rate $\left(F_{(1,12)}=14.55, p=0.002, \eta^{2}=0.548\right)$, not for false alarm rate $\left(F_{(1,12)}=2.54, p=0.137, \eta^{2}=0.175\right)$. In addition, we evaluated the so-tDCS effect on visual memory without correcting for sleepiness in the analysis (rmANOVA). Here, improved retention performance after so-tDCS compared with sham was observed, which, however, failed to reach signifi- 
cance $\left(F_{(1,14)}=3.58, p=0.079, \eta^{2}=\right.$ $0.204)$. With regard to main effects of stimulation and time point on visual recognition performance, no significant effects were evident, but a trend for a main effect of session emerged (stimulation, $\left.F_{(1,12)}=3.80, p=0.075\right)$.

Location retrieval performance was not affected by so-tDCS (stimulation $X$ time point interaction: $F_{(1,12)}=0.01, p=$ $0.913, \eta^{2}=0.001$; performance change across nap in \%: so-tDCS, 8.37, 95\% CI: -16.62 to -0.12 ; sham, $-4.95,95 \% \mathrm{CI}$ : -16.55 to 6.65$)$. Only a decline in spatial memory after the nap independent of stimulation condition was found (pre- to post-nap memory change; main effect time point: $F_{(1,12)}=6.18, p=0.029$; pre-nap in $\%:-12.87,95 \%$ CI: -35.84 to 10.10 , postnap in $\%:-19.53,95 \% \mathrm{CI}:-39.50$ to 0.44$)$.

For comparison with previous studies, we also examined so-tDCS effects on retention performance in the word pairedassociate learning task (verbal memory). We observed no so-tDCS effect on cued recall performance (statistically controlling for sleepiness, stimulation $X$ time point interaction: $F_{(1,12)}=2.27$, $\left.p=0.156, \eta^{2}=0.149, n=15\right)$, but MCI patients showed a significant decrement of recall performance independent of stimulation condition from before to after the nap (main effect time point: $F_{(1,12)}=12.96, p=0.003$; pre-nap in $\%: 45.9 \pm 6.6$; post-nap in $\%: 39.1 \pm 6.1$ ). Baseline verbal memory performance before napping did not differ between so-tDCS and sham session $\left(t_{(14)}=1.03, p=0.322\right)$ and no ceiling effect was observable (mean pre-nap percentage correct scores: sotDCS, 50.5, 95\% CI: 35.4-65.6; sham, 46.3, 95\% CI: 33.0-59.7).

To determine the specificity of so-tDCS effects, a procedural memory task was assessed in addition to declarative memory tasks. Neither main nor interaction effects were noted (all $p>0.5)$.

In sum, so-tDCS effects on the behavioral level revealed beneficial effects on the recognition performance of pictures after correcting for the confounding variable sleepiness. No effects of so-tDCS were seen on the location memory subtask, the verbal memory task, or the procedural memory task.

\section{Relationship between effects on sleep measures and memory performance}

To examine the relationship between stimulation-induced changes in sleep measures ( $\mathrm{SO}$ and spindle power changes as well as sleep stages) and visual memory performance changes, we correlated differences in sleep measures between stimulation conditions with the difference in visual recognition (pre- to post-nap) performance change between so-tDCS and sham condition. For power measures as well as sleep stages, no significant correlations were found (all $p>0.3$ ).

Finally, we investigated whether coupling between centroparietal SO and fast spindles, measured by the SI, was related to visual task performance change. We found that a positive "postnap - pre-nap" task performance difference tended to be associated with synchronized SO-to-fast-spindle PAC in so-tDCS condition (SI angles close to 0), whereas no such relationship could be observed for the sham condition (Fig. $5 a$ ).

Considering the relationship between stimulation-induced changes in PAC and visual recognition performance, an improvement in memory performance due to so-tDCS tended to accompany stronger synchronization (SI phase) between SO and fast spindle power when taking both phase differences (SI angles) and locking strengths (SI radii) into account (Fig. 5b). Fast spindle power during the SO up-phase (after the event-centering downphase) has been found previously to correlate with (verbal) memory performance (Ngo et al., 2013); therefore, we additionally applied a very similar measure (fast spindle power peak and its timing during that SO up-phase). We found no correlation of the fast spindle power peak value or its timing (absolute time difference to SO-up phase) with visual task performance change due to stimulation (and within condition, all Pearson's $|r|<0.27$, all $p>0.4)$.

To summarize this section, stimulation-induced improvement of visual memory did not correlate significantly with changes in overall SO or spindle power, but rather was associated with enhanced synchronization between $\mathrm{SO}$ and fast spindle power fluctuations.

\section{Discussion}

In light of the mounting evidence for the association between sleep disruptions and memory decline in MCI, we investigated whether specific memory-relevant sleep characteristics-in particular, SO, fast spindles, and the coupling between these oscillations - could be enhanced by so-tDCS during a daytime nap in MCI patients. Moreover, we intended to promote visual memory consolidation via stimulation in this patient group. We showed, for the first time in MCI patients, that so-tDCS significantly in- 
creased SO and spindle power. Importantly, we demonstrated that so-tDCS led to an enhanced endogenous SO-to-spindle coupling in the following way: spindle power was significantly amplified during the depolarizing SO up-phases and synchronization between SO and the fast spindle power signal was stronger. In addition, we found an improvement in visual memory performance that tended to be associated with stronger synchronization between $\mathrm{SO}$ and fast spindle power.

\section{so-tDCS increases SO and spindle activity as well as their functional coupling in MCI patients}

The impact of so-tDCS on SO power in the present study is consistent with previous studies examining the effects of so-tDCS during sleep on memory-relevant sleep measures in healthy older (during night time sleep: Paßmann et al., 2016; during daytime nap: Westerberg et al., 2015; Ladenbauer et al., 2016) and healthy young adults (during night time sleep: Marshall et al., 2006). Our observation of amplified fast spindle activity over frontal and centroparietal sites is consistent with previous results from our group in older adults (Ladenbauer et al., 2016; Paßmann et al., 2016) but contrary to those in healthy older (Westerberg et al., 2015) and healthy young individuals (Marshall et al., 2006). A possible reason for this discrepancy may be the variation in stimulation procedure because studies from our group controlled for ongoing sleep (sleep stages 2, 3, or 4) before every stimulation block (state-dependent stimulation, but see Westerberg et al., 2015). Since stimulation effects strongly depend on ongoing brain state (Marshall et al., 2011) a sleep-state-dependent protocol of this kind may be critical, specifically for older adults given their increased sleep fragmentation (Bliwise et al., 2009), an issue potentiated in patients with neurodegenerative disease (Lim et al., 2013).

A plausible mechanism for the poststimulation EEG effects could be entrainment of endogenous activity outlasting the acute stimulation. Externally applied slow oscillatory currents that produce electric fields as low as $0.5-1 \mathrm{~V} / \mathrm{m}$ in magnitude (comparable to human so-tDCS studies) were sufficient to entrain oscillatory brain activity acutely in rats (Ozen et al., 2010; Binder et al., 2014). These effects did not persist after stimulation cessation (Ozen et al., 2010) or after-effects failed to reach significance (Binder et al., 2014). However, this finding may be attributed to the short stimulation duration in the range of seconds (maximum $60 \mathrm{~s}$ in Ozen et al., 2010) compared with human studies (5-30 $\mathrm{min}$ ), with after-effects being more pronounced after longer stimulation (Strüber et al., 2015; Campos-Beltrán and Marshall, 2017).

Considering the coupling between SO and spindles we observed in both conditions that SO modulated fast spindle power such that it exhibited up- and down-phases similar to SO on average, with positive peaks occurring during the depolarizing SO up-phase close to (but slightly before) the SO peak (cf. Fig. $3 c$ ). These stimulation-independent observations are well in line with previous results (Mölle et al., 2011, 2002; Niknazar et al., 2015; Staresina et al., 2015). Furthermore, synchronization between SO and fast spindle power in sham condition as quantified by subject-averaged synchronization indices is comparable to what was reported in Staresina et al. (2015) ( V = 6.84 here vs V = 6.55 there), who demonstrated coupling between SO and spindles as well as between spindle and ripple activity in a hierarchical manner in humans. Notably, we provided evidence for a strong enhancement of SO-to-spindle coupling by so-tDCS - quantified by both fast spindle power during SO up-phases and synchronization (cf. Fig. $3 b-d$ ). This is a particularly promising result in the light of accumulating evidence for the functional role of that specific coupling (Ruch et al., 2012; Ngo et al., 2013; Niknazar et al., 2015).

\section{so-tDCS improves visual memory in MCI patients}

We found that so-tDCS led to improved visual recognition performance, consistent with our previous study on healthy older individuals (Ladenbauer et al., 2016). Regarding location memory (retrieval of picture locations) and verbal memory (word pair task) we found no effect of so-tDCS in MCI patients, similar to the results in healthy older adults (Ladenbauer et al., 2016). For the spatial subtask this may be due to the small number of valid items (retrieval of location only if participant recognized the picture as "known") or the difficulty of the task for our participants in the light of recent evidence for beneficial so-tDCS effects primarily on consolidation of relatively simple information (Barham et al., 2016). For the verbal task relatively strong semantic associations in our word pairs may have decreased the sensitivity of this task to detect stimulation-related memory effects, since previous evidence indicated higher sleep-dependent benefits for weak or no semantic associations (Drosopoulos et al., 2007, Lo et al., 2014), especially in nap sleep (Lo et al., 2014). This may explain why we did not find effects on associative verbal memory in contrast to previous studies (Marshall et al., 2006; Westerberg et al., 2015).

\section{Relationship between changes of sleep characteristics and visual memory improvement}

Changes in overall SO or spindle power as well as sleep stage 2 proportion were not significantly correlated with visual performance change, similar to previous so-tDCS studies that could not show a correlation between changes in these parameters (Marshall et al., 2006; Prehn-Kristensen et al., 2014; Del Felice et al., 2015; Westerberg et al., 2015; Ladenbauer et al., 2016). This may be attributable to low statistical power because of small sample sizes. Each of these oscillations, SO and spindles, on their own (without intervention) have been correlated with overnight retention of memories (e.g., Schabus et al., 2004; Tamminen et al., 2010; Mander et al., 2014), however, a recent study with large sample size $(n=929)$ challenges these results (Ackermann et al., 2015). It is well likely that the functional coupling between SO and spindles plays a more dominant role for memory consolidation. From our results, stimulation-induced improvement in visual memory is predictable by enhanced synchronization between SO and fast spindle power (Fig. 5b). Nevertheless, it should be noted that in-phase synchrony (SI angles $=0$ ) may not necessarily be the optimal relationship for memory consolidation compared with a (small) phase difference instead (e.g., fast spindle power peak shortly before SO peak; see Cox et al., 2014; Staresina et al., 2015). Future studies should determine whether stronger SO-to-spindle coupling (in synchrony or locked with a particular phase shift) consistently leads to improved consolidation during sleep. Moreover, despite accumulating evidence indicating a strong relevance of SO-modulation by so-tDCS for memory improvement, such as the specificity of stimulation frequency (Marshall et al., 2011), brain state (Kirov et al., 2009; Marshall et al., 2011), shape of the so-tDCS pulse (Sahlem et al., 2015), and stimulation montage (Garside et al., 2015), memory effects may also be due to changes in synaptic plasticity independent of oscillatory activity (Vossen et al., 2015), an issue to be addressed in the future.

Our results are very encouraging in the light of strong evidence for sleep deteriorations and their consequences on cogni- 
tive functions in MCI: sleep disturbances are associated with a decline in memory consolidation (Westerberg et al., 2012), facilitate accumulation of amyloid- $\beta$ (Kang et al., 2009), and may thereby trigger the pathophysiological process leading to $\mathrm{AD}$ (Ju et al., 2014; Mander et al., 2016). Therefore, optimizing specific sleep parameters by so-tDCS may constitute a promising therapeutic approach in two respects. First, enhancement of memoryrelevant sleep parameters (SO, spindles, and their functional coupling) can lead to improved memory consolidation, which helps to preserve cognitive abilities and possibly reduces clinical severity in AD (Ju et al., 2014). Second, improvement of sleep physiology, in particular $\mathrm{SO}$ activity, may decelerate the progression of disease pathology in MCI patients through enhanced clearance of amyloid- $\beta$ (Xie et al., 2013). In these regards, sotDCS may be more effective compared with pharmacological treatments, which so far could not enhance both $\mathrm{SO}$ activity and functional SO-to-spindle coupling during sleep at the same time (Feld et al., 2013; Niknazar et al., 2015) and which failed to improve, or even decreased, memory performance (Vienne et al., 2012; Feld et al., 2013; Hall-Porter et al., 2014) with one exception (Niknazar et al., 2015).

\section{Limitations and conclusion}

A few limitations of the study should be noted: First, patients tended to feel sleepier following a nap with so-tDCS compared with the sham condition. This effect on sleepiness was also found in healthy older individuals in our previous study (which was significant), whereas no other study applying so-tDCS during sleep reported this effect before. We cannot rule out that increased SO activity due to so-tDCS persisted until the survey after the nap (Nitsche and Paulus, 2001) although it is very unlikely. Note, however, that we controlled statistically for sleepiness to exclude its potential effect on memory performance. Second, to analyze the immediate effects of so-tDCS on coupling between $\mathrm{SO}$ and spindles, a rather limited amount of EEG data (from up to five $1 \mathrm{~min}$ stimulation-free intervals) could be used due to the study design. This explains why the power patterns in the TFRs, despite strong similarities, do not emerge as clearly as in methodologically related studies, particularly in the slow spindle frequency range (Mölle et al., 2011; Staresina et al., 2015). However, the overall agreements of our results with previous ones support the validity of these analyses. Furthermore, we observed that visual recognition performance varied stronger across MCI patients in the sham compared with the so-tDSC session. Note, however, that the study was balanced with respect to so-tDCS/ sham order as well as task versions and no other difference than stimulation condition occurred between sessions. In addition, although we observed medium to large effect sizes for EEG power changes due to so-tDCS (Cohen's $d=0.5-1.16$ ), effects on visual recognition memory were relatively small $\left(\eta^{2}=0.308\right)$. One explanation for these findings might be that MCI patients need prolonged so-tDCS phases, more iterations, and/or repeated sessions to induce more pronounced memory effects. Further, although SO, spindles, and their temporal coordination seem necessary for memory consolidation, other neurobiological processes (e.g., BDNF release; Fritsch et al., 2010) probably contribute as well and may not be modulated by so-tDCS. Another limitation of our study is the rather small sample size, which might explain the lack of significant correlations between EEG and memory effects.

Despite these limitations, our results clearly demonstrate the potential of so-tDCS to enhance functionally relevant sleep parameters in $\mathrm{MCI}$, including for the first time the coupling of SO to spindle activity, a mechanistic component considered crucial for the transfer of memories from hippocampus to cortical longterm storage networks. Apart from benefits on memory consolidation, as shown here for visual recognition memory, the stimulation may thereby delay the progression of Alzheimer's pathology (Ju et al., 2014). Therefore, it would be worthwhile to assess the extent to which this noninvasive stimulation method can be optimized for individualized treatment, for example, by fine-tuning to each patient's sleep structure in an automated closed-loop manner (Ngo et al., 2013).

\section{References}

Ackermann S, Hartmann F, Papassotiropoulos A, de Quervain DJ, Rasch B (2015) No associations between interindividual differences in sleep parameters and episodic memory consolidation. Sleep 38:951-959. Medline

Algarabel S, Fuentes M, Escudero J, Pitarque A, Peset V, Mazón JF, Meléndez JC (2012) Recognition memory deficits in mild cognitive impairment. Aging, Neuropsychol Cogn 19:608-619. CrossRef Medline

Antonenko D, Diekelmann S, Olsen C, Born J, Mölle M (2013) Napping to renew learning capacity: enhanced encoding after stimulation of sleep slow oscillations. Eur J Neurosci 37:1142-1151. CrossRef Medline

Aschenbrenner S, Tucha O, Lange K (2000) Regensburg word fluency test. Göttingen, Germany: Hofgrefe.

Bäckman L, Jones S, Berger AK, Laukka EJ, Small BJ (2004) Multiple cognitive deficits during the transition to Alzheimer's disease. J Intern Med 256:195-204. CrossRef Medline

Barbeau E, Didic M, Tramoni E, Felician O, Joubert S, Sontheimer A, Ceccaldi M, Poncet M (2004) Evaluation of visual recognition memory in MCI patients. Neurology 62:1317-1322. CrossRef Medline

Barham MP, Enticott PG, Conduit R, Lum JA (2016) Transcranial electrical stimulation during sleep enhances declarative (but not procedural) memory consolidation: evidence from a meta-analysis. Neurosci Biobehav Rev 63:65-77. CrossRef Medline

Benjamini Y, Hochberg Y (1995) Controlling the false discovery rate: a practical and powerful approach to multiple testing. J R Stat Soc B 57: 289-300.

Bennett IJ, Golob EJ, Parker ES, Starr A (2006) Memory evaluation in mild cognitive impairment using recall and recognition tests. J Clin Exp Neuropsychol 28:1408-1422. CrossRef Medline

Berens P (2009) CircStat: a MATLAB toolbox for circular statistics. Journal of Statistical Software 31:1-21.

Bes F, Wilde-Frenz J, Schulz H (1992) Application of the Tiredness Symptoms Scale during sleep deprivation. Sleep-Wake Research in The Netherlands 3:19-20.

Besson G, Ceccaldi M, Tramoni E, Felician O, Didic M, Barbeau EJ (2015) Fast, but not slow, familiarity is preserved in patients with amnestic mild cognitive impairment. Cortex 65:36-49. CrossRef Medline

Binder S, Berg K, Gasca F, Lafon B, Parra LC, Born J, Marshall L (2014) Transcranial slow oscillation stimulation during sleep enhances memory consolidation in rats. Brain Stimul 7:508-515. CrossRef Medline

Bliwise DL, Foley DJ, Vitiello MV, Ansari FP, Ancoli-Israel S, Walsh JK (2009) Nocturia and disturbed sleep in the elderly. Sleep Med 10:540548. CrossRef Medline

Buckelmüller J, Landolt HP, Stassen HH, Achermann P (2006) Trait-like individual differences in the human sleep electroencephalogram. Neuroscience 138:351-356. CrossRef Medline

Buysse DJ, Reynolds CF 3rd, Monk TH, Berman SR, Kupfer DJ (1989) The Pittsburgh Sleep Quality Index: a new instrument for psychiatric practice and research. Psychiatry Res 28:193-213. Medline

Campos-Beltrán D, Marshall L (2017) Electric stimulation to improve memory consolidation during sleep. In: Cognitive neuroscience of memory consolidation, pp 301-312. CrossRef

Clemens Z, Mölle M, Eross L, Barsi P, Halász P, Born J (2007) Temporal coupling of parahippocampal ripples, sleep spindles and slow oscillations in humans. Brain 130:2868-2878. CrossRef Medline

Cohen MX (2008) Assessing transient cross-frequency coupling in EEG data. J Neurosci Methods 168:494-499. CrossRef Medline

Cox R, van Driel J, de Boer M, Talamini LM (2014) Slow oscillations during sleep coordinate interregional communication in cortical networks. J Neurosci 34:16890-16901. CrossRef Medline

Del Felice A, Magalini A, Masiero S (2015) Slow-oscillatory transcranial di- 
rect current stimulation modulates memory in temporal lobe epilepsy by altering sleep spindle generators: a possible rehabilitation tool. Brain Stimul 8:567-573. CrossRef Medline

Diekelmann S, Born J (2010) The memory function of sleep. Nat Rev Neurosci 11:114-126. CrossRef Medline

Drosopoulos S, Schulze C, Fischer S, Born J (2007) Sleep's function in the spontaneous recovery and consolidation of memories. J Exp Psychol Gen 136:169-183. CrossRef Medline

Dudas RB, Clague F, Thompson SA, Graham KS, Hodges JR (2005) Episodic and semantic memory in mild cognitive impairment. Neuropsychologia 43:1266-1276. CrossRef Medline

Eggert T, Dorn H, Sauter C, Nitsche MA, Bajbouj M, Danker-Hopfe H (2013) No effects of slow oscillatory transcranial direct current stimulation (tDCS) on sleep-dependent memory consolidation in healthy elderly subjects. Brain Stimul 6:938-945. CrossRef Medline

Feld GB, Wilhelm I, Ma Y, Groch S, Binkofski F, Mölle M, Born J (2013) Slow wave sleep induced by GABA agonist tiagabine fails to benefit memory consolidation. Sleep 36:1317-1326. CrossRef Medline

Fritsch B, Reis J, Martinowich K, Schambra HM, Ji Y, Cohen LG, Lu B (2010) Direct current stimulation promotes BDNF-dependent synaptic plasticity: potential implications for motor learning. Neuron 66:198-204. CrossRef Medline

Frohnhofen H, Bibl M, Nickel B, Popp R (2010) Der Essener Fragebogen Alter und Schläfrigkeit (EFAS): ein neues Assessmentinstrument zur Messung von Tagesschläfrigkeit bei alten Menschen. Eur J Ger 12:84-89.

Garside P, Arizpe J, Lau CI, Goh C, Walsh V (2015) Cross-hemispheric alternating current stimulation during a nap disrupts slow wave activity and associated memory consolidation. Brain Stimul 8:520-527. CrossRef Medline

Gatterer G (2008) Alters-Konzentrations-Test (AKT), Ed 2. Göttingen, Germany: Hofgrefe.

Griefahn B, Künemund P, Bröde P, Mehnert P (2001) Zur Validität der deutschen Übersetzung des Morningness-Eveningness-Questionnaires von Horne und Östberg. Somnologie 5:71-80.

Hall-Porter JM, Schweitzer PK, Eisenstein RD, Ahmed HA, Walsh JK (2014) The effect of two benzodiazepine receptor agonist hypnotics on sleepdependent memory consolidation. J Clin Sleep Med 10:27-34. Medline

Hardy J, Selkoe DJ (2002) The amyloid hypothesis of Alzheimer's disease: progress and problems on the road to therapeutics. Science 297:353-356. CrossRef Medline

Hedges LV (2007) Effect sizes in cluster-randomized designs. J Educ Behav Stat 32:341-370. CrossRef

Helmstaedter, C, Lendt, M, Lux, S, (2001) Verbaler Lern- und Merkfähigkeitstest. Göttingen, Germany: Beltz.

Jessen F, Wolfsgruber S, Wiese B, Bickel H, Mösch E, Kaduszkiewicz H, Pentzek M, Riedel-Heller SG, Luck T, Fuchs A, Weyerer S, Werle J, van den Bussche H, Scherer M, Maier W, Wagner M; German Study on Aging, Cognition and Dementia in Primary Care Patients (2014) AD dementia risk in late $\mathrm{MCI}$, in early $\mathrm{MCI}$, and in subjective memory impairment. Alzheimers Dement 10:76-83. CrossRef Medline

Johns MW (1991) A new method for measuring daytime sleepiness: the Epworth sleepiness scale. Sleep 14:540-545. CrossRef Medline

Ju YE, Lucey BP, Holtzman DM (2014) Sleep and Alzheimer disease pathology: a bidirectional relationship. Nat Rev Neurol 10:115-119. CrossRef Medline

Kang JE, Lim MM, Bateman RJ, Lee JJ, Smyth LP, Cirrito JR, Fujiki N, Nishino S, Holtzman DM (2009) Amyloid- $\beta$ dynamics are regulated by orexin and the sleep-wake cycle. Science 326:1005-1007. CrossRef Medline

Kirov R, Weiss C, Siebner HR, Born J, Marshall L (2009) Slow oscillation electrical brain stimulation during waking promotes EEG theta activity. Proc Natl Acad Sci U S A 106:15460-15465. CrossRef Medline

Kühner C, Bürger C, Keller F, Hautzinger M (2007) Reliability and validity of the Revised Beck Depression Inventory (BDI-II). Nervenarzt 78:651656. CrossRef Medline

Ladenbauer J, Külzow N, Passmann S, Antonenko D, Grittner U, Tamm S, Flöel A (2016) Brain stimulation during an afternoon nap boosts slow oscillatory activity and memory consolidation in older adults. Neuroimage 142:311-323. CrossRef Medline

Lang PJ, Bradley MM, Cuthbert BN (1999) International affective picture system (IAPS): affective ratings of pictures and instruction manual. Gainesville, FL: Center for Research in Psychophysiology.

Lim AS, Kowgier M, Yu L, Buchman AS, Bennett DA (2013) Sleep fragmen- tation and the risk of incident Alzheimer's Disease and cognitive decline in older persons. Sleep 36:1027-1032. CrossRef Medline

Lo JC, Dijk DJ, Groeger JA (2014) Comparing the effects of nocturnal sleep and daytime napping on declarative memory consolidation. PLoS One 9:e108100. CrossRef Medline

Luria RE (1975) The validity and reliability of the visual analogue mood scale. J Psychiatr Res 12:51-57. CrossRef Medline

Mander BA, Rao V, Lu B, Saletin JM, Lindquist JR, Ancoli-Israel S, Jagust W, Walker MP (2013) Prefrontal atrophy, disrupted NREM slow waves and impaired hippocampal-dependent memory in aging. Nat Neurosci 16: 357-364. CrossRef Medline

Mander BA, Rao V, Lu B, Saletin JM, Ancoli-Israel S, Jagust WJ, Walker MP (2014) Impaired prefrontal sleep spindle regulation of hippocampaldependent learning in older adults. Cereb Cortex 24:3301-3309. CrossRef Medline

Mander BA, Winer JR, Jagust WJ, Walker MP (2016) Sleep: a novel mechanistic pathway, biomarker, and treatment target in the pathology of Alzheimer's Disease? Trends Neurosci 39:552-566. CrossRef Medline

Mandler G (2008) Familiarity breeds attempts: a critical review of dualprocess theories of recognition. Perspect Psychol Sci 3:390-399. CrossRef Medline

Maris E, Oostenveld R (2007) Nonparametric statistical testing of EEG- and MEG-data. J Neurosci Methods 164:177-190. CrossRef Medline

Marshall L, Helgadóttir H, Mölle M, Born J (2006) Boosting slow oscillations during sleep potentiates memory. Nature 444:610-613. CrossRef Medline

Marshall L, Kirov R, Brade J, Mölle M, Born J (2011) Transcranial electrical currents to probe EEG brain rhythms and memory consolidation during sleep in humans. PLoS One 6:e16905. CrossRef Medline

Migo E, Montaldi D, Norman KA, Quamme J, Mayes A (2009) The contribution of familiarity to recognition memory is a function of test format when using similar foils. Q J Exp Psychol (Hove) 62:1198-1215. CrossRef Medline

Mölle M, Marshall L, Gais S, Born J (2002) Grouping of spindle activity during slow oscillations in human non-rapid eye movement sleep. J Neurosci 22:10941-10947. Medline

Mölle M, Bergmann TO, Marshall L, Born J (2011) Fast and slow spindles during the sleep slow oscillation: disparate coalescence and engagement in memory processing. Sleep 34:1411-1421. CrossRef Medline

Nelson DL, McEvoy CL, Schreiber TA (2004) The University of South Florida free association, rhyme, and word fragment norms. Behav Res Methods Instrum Comput 36:402-407. CrossRef Medline

Ngo HV, Martinetz T, Born J, Mölle M (2013) Auditory closed-loop stimulation of the sleep slow oscillation enhances memory. Neuron 78:545553. CrossRef Medline

Niknazar M, Krishnan GP, Bazhenov M, Mednick SC (2015) Coupling of thalamocortical sleep oscillations are important for memory consolidation in humans. PLoS One 10:e0144720. CrossRef Medline

Nitsche MA, Paulus W (2001) Sustained excitability elevations induced by transcranial DC motor cortex stimulation in humans. Neurology 57: 1899-1901. CrossRef Medline

Oostenveld R, Fries P, Maris E, Schoffelen JM (2011) FieldTrip: open source software for advanced analysis of MEG, EEG, and invasive electrophysiological data. Comput Intell Neurosci 2011:1. CrossRef Medline

Ozen S, Sirota A, Belluscio MA, Anastassiou CA, Stark E, Koch C, Buzsáki G (2010) Transcranial electric stimulation entrains cortical neuronal populations in rats. J Neurosci 30:11476-11485. CrossRef Medline

Paßmann S, Külzow N, Ladenbauer J, Antonenko D, Grittner U, Tamm S, Flöel A (2016) Boosting slow oscillatory activity using tDCS during early nocturnal slow wave sleep does not improve memory consolidation in healthy older adults. Brain Stimul 9:730-739. CrossRef Medline

Prehn-Kristensen A, Munz M, Göder R, Wilhelm I, Korr K, Vahl W, Wiesner CD, Baving L (2014) Transcranial oscillatory direct current stimulation during sleep improves declarative memory consolidation in children with attention-deficit/hyperactivity disorder to a level comparable to healthy controls. Brain Stimul 7:793-799. CrossRef Medline

Prinz PN, Vitaliano PP, Vitiello MV, Bokan J, Raskind M, Peskind E, Gerber C (1982) Sleep, EEG and mental function changes in senile dementia of the Alzheimer's type. Neurobiol Aging 3:361-370. CrossRef Medline

Rasch B, Born J (2013) About sleep's role in memory. Physiol Rev 93:681766. CrossRef Medline

Rechtschaffen, A, Kales, A, (1968) A manual of standardized terminology, 
techniques and scoring system for sleep stages of human subjects. Washington, D.C.: U.S. Government Printing Office.

Ruch S, Markes O, Duss SB, Oppliger D, Reber TP, Koenig T, Mathis J, Roth C, Henke K (2012) Sleep stage II contributes to the consolidation of declarative memories. Neuropsychologia 50:2389-2396. CrossRef Medline

Sahlem GL, Badran BW, Halford JJ, Williams NR, Korte JE, Leslie K, Strachan M, Breedlove JL, Runion J, Bachman DL, Uhde TW, Borckardt JJ, George MS (2015) Oscillating square wave Transcranial Direct Current Stimulation (tDCS) delivered during slow wave sleep does not improve declarative memory more than sham: A randomized sham controlled crossover study. Brain Stimul 8:528-534. CrossRef Medline

Schabus M, Gruber G, Parapatics S, Sauter C, Klösch G, Anderer P, Klimesch W, Saletu B, Zeitlhofer J (2004) Sleep spindles and their significance for declarative memory consolidation. Sleep 27:1479-1485. CrossRef Medline

Schneider TR, Engel AK, Debener S (2008) Multisensory identification of natural objects in a two-way crossmodal priming paradigm. Exp Psychol 55:121-132. CrossRef Medline

Sperling RA, Aisen PS, Beckett LA, Bennett DA, Craft S, Fagan AM, Iwatsubo T, Jack CR Jr, Kaye J, Montine TJ, Park DC, Reiman EM, Rowe CC, Siemers E, Stern Y, Yaffe K, Carrillo MC, Thies B, Morrison-Bogorad M, Wagster MV, Phelps CH (2011) Toward defining the preclinical stages of Alzheimer's disease: Recommendations from the National Institute on Aging-Alzheimer's Association workgroups on diagnostic guidelines for Alzheimer's disease. Alzheimers Dement 7:280-292. CrossRef Medline

Staresina BP, Bergmann TO, Bonnefond M, van der Meij R, Jensen O, Deuker L, Elger CE, Axmacher N, Fell J (2015) Hierarchical nesting of slow oscillations, spindles and ripples in the human hippocampus during sleep. Nat Neurosci 18:1679-1686. CrossRef Medline

Strüber D, Rach S, Neuling T, Herrmann CS (2015) On the possible role of stimulation duration for after-effects of transcranial alternating current stimulation. Front Cell Neurosci 9:311. CrossRef Medline

Tamminen J, Payne JD, Stickgold R, Wamsley EJ, Gaskell MG (2010) Sleep spindle activity is associated with the integration of new memories and existing knowledge. J Neurosci 30:14356-14360. Medline

Tombaugh TN (2004) Trail Making Test A and B: Normative data stratified by age and education. Arch Clin Neuropsychol 19:203-214. CrossRef Medline

Tucker AM, Dinges DF, Van Dongen HP (2007) Trait interindividual differences in the sleep physiology of healthy young adults. J Sleep Res 16: 170-180. CrossRef Medline

Van der Elst W, Van Boxtel MP, Van Breukelen GJ, Jolles J (2006) The Stroop color-word test: influence of age, sex, and education; and norma- tive data for a large sample across the adult age range. Assessment 13:6279. CrossRef Medline

Verbeke G, Molenberghs G (2000) Linear mixed models for longitudinal data. New York: Springer.

Vienne J, Lecciso G, Constantinescu I, Schwartz S, Franken P, Heinzer R, Tafti M (2012) Differential effects of sodium oxybate and baclofen on EEG, sleep, neurobehavioral performance, and memory. Sleep 35:1071-1083. CrossRef Medline

Vossen A, Gross J, Thut G (2015) Alpha power increase after transcranial alternating current stimulation at alpha frequency (a-tACS) reflects plastic changes rather than entrainment. Brain Stimul 8:499-508. CrossRef Medline

Walker MP, Brakefield T, Morgan A, Hobson JA, Stickgold R (2002) Practice with sleep makes perfect: sleep-dependent motor skill learning. Neuron 35:205-211. CrossRef Medline

Wang G, Grone B, Colas D, Appelbaum L, Mourrain P (2011) Synaptic plasticity in sleep: learning, homeostasis and disease. Trends Neurosci 34:452-463. CrossRef Medline

Watson D, Clark LA, Tellegen A (1988) Development and validation of brief measures of positive and negative affect: the PANAS scales. J Pers Soc Psychol 54:1063-1070. CrossRef Medline

Wechsler D (1997) Wechsler adult intelligence scale, Ed 3. San Antonio, TX: Psychological Corporation.

Westerberg CE, Mander BA, Florczak SM, Weintraub S, Mesulam MM, Zee PC, Paller KA (2012) Concurrent impairments in sleep and memory in amnestic mild cognitive impairment. J Int Neuropsychol Soc 18:490-500. CrossRef Medline

Westerberg CE, Florczak SM, Weintraub S, Mesulam MM, Marshall L, Zee PC, Paller KA (2015) Memory improvement via slow-oscillatory stimulation during sleep in older adults. Neurobiol Aging 36:2577-2586. CrossRef Medline

Wolk DA, Signoff ED, Dekosky ST (2008) Recollection and familiarity in amnestic mild cognitive impairment: a global decline in recognition memory. Neuropsychologia 46:1965-1978. Medline

Wolk DA, Mancuso L, Kliot D, Arnold SE, Dickerson BC (2013) Familiaritybased memory as an early cognitive marker of preclinical and prodromal AD. Neuropsychologia 51:1094-1102. CrossRef Medline

Xie L, Kang H, Xu Q, Chen MJ, Liao Y, Thiyagarajan M, O’Donnell J, Christensen DJ, Nicholson C, Iliff JJ, Takano T, Deane R, Nedergaard M (2013) Sleep drives metabolite clearance from the adult brain. Science 342:373-377. CrossRef Medline

Zimmermann P, Fimm B (1995) Test for attentional performance (TAP). Herzogenrath, Germany: PsyTest. 